\title{
Coarse-Grained Kinetic Computations for Rare Events: Application to Micelle Formation
}

\author{
Dmitry I. Kopelevich $*$ Athanassios Z. Panagiotopoulos $₫$ and Ioannis G. Kevrekidid \\ Department of Chemical Engineering \\ Princeton University \\ Princeton, NJ 08544
}

\begin{abstract}
We discuss a coarse-grained approach to the computation of rare events in the context of grand canonical Monte Carlo (GCMC) simulations of self-assembly of surfactant molecules into micelles. The basic assumption is that the computational system dynamics can be decomposed into two parts - fast (noise) and slow (reaction coordinates) dynamics, so that the system can be described by an effective, coarse grained Fokker-Planck (FP) equation. While such an assumption may be valid in many circumstances, an explicit form of FP equation is not always available. In our computations we bypass the analytic derivation of such an effective FP equation. The effective free energy gradient and the state-dependent magnitude of the random noise, which are necessary to formulate the effective Fokker-Planck equation, are obtained from ensembles of short bursts of microscopic simulations with judiciously chosen initial conditions. The reaction coordinate in our micelle formation problem is taken to be the size of a cluster of surfactant molecules. We test the validity of the effective FP description in this system and reconstruct a coarse-grained free energy surface in good agreement with full-scale GCMC simulations. We also show that, for very small clusters, the cluster size seizes to be a good reaction coordinate for a one-dimensional effective description. We discuss possible ways to improve the current model and to take higher-dimensional coarse-grained dynamics into account.
\end{abstract}




\section{INTRODUCTION}

The development of efficient computational methods for the study of rare events is a subject of intense current interest and research across many disciplines 1,2.3.4.5.6 . Direct microscopic (e.g., molecular dynamics or kinetic Monte Carlo) simulations of rare events can be extremely time-consuming since most of the computational time is spent sampling the free energy surface close to local free energy minima and the transition states are sampled only during an exponentially small fraction of the simulation time.

Many systems can be successfully described through a low-dimensional effective freeenergy surface in terms of dynamically meaningful observable quantities (often referred to as "reaction coordinates", see for example the discussion in Ref. 7). In such cases it is reasonable to expect that the evolution of the probability density of the variables that parameterize this surface may be described in terms of an effective Fokker-Planck (FP) equation. The deterministic part of the FP equation will then contain the gradient of the effective free energy surface with respect to the few "coarse" variables (reaction coordinates, "observables") chosen to parameterize it, as well as the local diffusivity of the expected motion.

In many cases of practical interest, this effective FP equation is not available in closed form. Recently, Hummer and Kevrekidis ${ }^{7}$ have proposed a so called kinetic approach that bypasses the analytical derivation of such an equation, and uses the concept of its existence to guide the detailed (molecular dynamics, Monte Carlo) simulations. In this approach, the components of the effective FP equation are estimated through multiple, relatively short microscopic simulations with judiciously chosen initial conditions.

In this paper, we apply this kinetic approach to Monte Carlo (MC) simulations of selfassembly of surfactant molecules into micelles. We consider a lattice model ${ }^{8.9}$ with only short-range hydrophobic interactions between the molecules. The "dynamics" of these simulations are artificial; the kinetic approach allows us to explore the free energy surface using this artificial dynamic evolution. Indeed, in section $\mathrm{VC}$ we show that the free energy surface predicted by the kinetic approach does not depend on a particular choice of the MC "dynamics". Moreover, we expect that the methods and conclusions of this work can also be applied to real-time, (molecular-dynamic) simulations of self-assembly.

The assumption of an effective-FP (and associated Langevin) dynamics of micelle forma- 
tion is a departure from the usual assumption of the first order activated process of addition (removal) of single amphiphile molecules to (from) a micelle ${ }^{10.11}$. However, we show that the effective Langevin equation model performs well for the considered system; this suggests a link between the effective Langevin description and the master equation of the activated process model. This link needs to be investigated in the future.

In the companion paper ${ }^{12}$ (Paper I), we have considered "dynamics" of MC simulations of micelle formation and have discussed the application of the multiscale coarse projective integration and coarse Newton methods to these systems. We have observed that, as in the real physical dynamics, the rate-limiting step in the MC "dynamics" is the birth and death of micelles (as opposed to, e.g., altering of micelle shape and size as the external parameters are changed). In Paper I, we have used the first two moments of the micelle number density as our coarse variables (reaction coordinates, "observables"). In addition, we have performed the coarse computations of the system using a kinetic Monte Carlo (kMC) model for micelle birth and death with the rates obtained from the full-scale equilibrium simulation.

In the current paper, we compute the micelle formation rates directly from short-time MC simulations using the kinetic approach. The coarse variables here are the variables that characterize micelle size and shape. We assume that the coarse variables that can be used to parameterize a free energy surface description can be selected among physical attributes (such as size, energy, radius of gyration) of a cluster of surfactant molecules. Based on computational evidence supporting the existence of effectively one-dimensional long-term dynamics, we use a single coarse degree of freedom (a single "reaction coordinate", the cluster size) to parameterize the effective free energy surface and show that the remaining coarse degrees of freedom relax quickly to functions of (become slaved to) a single "master mode". We then demonstrate the validity of assumptions of the effective FP dynamics for the cluster size and estimate the effective free energy surface as well as the rates of micelle breakup, in good agreement with full equilibrium MC simulation.

We observe that the assumption of one-dimensional coarse dynamics breaks down for small cluster sizes and that, in order to successfully reconstruct the free energy surface, one needs to consider coarse-grained dynamics in at least a two-dimensional configuration space, where the second dimension can be chosen to be, e.g., the cluster energy.

The paper is organized as follows: In Section [II we state some basic results of the theory of stochastic processes, which form theoretical basis of the coarse kinetic approach. In 
Section III, we briefly review the lattice model and the Monte Carlo method used in our

simulations. We also present results of a long-time equilibrium simulation, which will be compared with the kinetic approach results in the subsequent sections. Section $\llbracket$ contains a detailed description of our implementation of the kinetic approach specific to simulations of micelle formation. Results of the kinetic approach calculations are reported in Section $\nabla$. In this section, we also check assumptions underlying the effective FP equation model for the micelle formation dynamics and validate these assumptions computationally. In Section VI we explore the micelle formation dynamics in the phase space parameterized by two coarse variables. We observe that, in most cases, the system quickly approaches an effective one-dimensional manifold -so that the one-dimensional FP model for the micelle formation dynamics is appropriate. We further observe that such a separation of timescales is much weaker for dynamics of small clusters. Finally, in Section VII we summarize our findings and briefly discuss some other coarse-grained, "equation-free" methods (coarse Newton and coarse reverse integration) and their application to the micelle formation problem.

\section{THEORETICAL BACKGROUND.}

In this section we review some standard results from the theory of stochastic processes and discuss their role in the kinetic approach. It is assumed here that the system dynamics can be described by a single coarse variable (reaction coordinate) $\psi(t)$. This assumption implies that all other variables quickly approach some sort of slow, attracting, one-dimensional manifold; that is, the statistics of the simulation quickly become functions of one observable; the slow manifold is the graph of this function. In our case of micelle formation, $\psi$ is chosen to be the size of a micelle, as measured by the number of amphiphile molecules contained in the micelle and it is assumed that all other physical attributes of a micelle (such as radii of gyration, density profile, energy, etc.) are quickly slaved (in an averaged sense) to its size. It will be shown in Section VI that this is a reasonable assumption.

Consider a general one-dimensional stochastic process $\psi(t)$. The evolution of the probability density $P(\psi, t)$ of $\psi$ obeys the following integral equation 13

$$
P(\psi, t+\tau)=\int \rho\left(\psi, t+\tau \mid \psi^{\prime}, t\right) P\left(\psi^{\prime}, t\right) d \psi^{\prime}
$$

where $\rho\left(\psi, t+\tau \mid \psi^{\prime}, t\right)$ is the transition probability from point $\psi^{\prime}$ at time $t$ to point $\psi$ at time 
$(t+\tau)$. The differential form of this equation, known as the Kramers-Moyal expansion, is as follows:

$$
\frac{\partial P(\psi, t)}{\partial t}=\sum_{n=1}^{\infty}\left(-\frac{\partial}{\partial \psi}\right)^{n} D^{(n)}(\psi, t) P(\psi, t)
$$

where

$$
D^{(n)}(\psi, t)=\left.\frac{1}{n !} \lim _{\tau \rightarrow 0} \frac{1}{\tau}\left\langle(\xi(t+\tau)-\xi(t))^{n}\right\rangle\right|_{\xi(t)=\psi}
$$

are the differential moments of the transition probability $\rho$. The angular brackets here denote ensemble averaging and $\xi$ denotes a realization of the stochastic process with a $\delta$-function distribution at the starting point $t, \xi(t)=\psi$.

This is a very general result and it applies to any one-dimensional stochastic process. If the process is Markovian and Gaussian, then only the first two terms in Eq. (2) are non-zero. Moreover, if the stochastic process is invariant with respect to the shift in time (which is true for the processes without external time-dependent forcing), then the expansion coefficients $D^{(n)}$ are independent of time. Hence, under these assumptions the stochastic process can be described by the Fokker-Planck equation 13

$$
\frac{\partial P(\psi, t)}{\partial t}=\left[-\frac{\partial}{\partial \psi} v(\psi)+\frac{\partial^{2}}{\partial \psi^{2}} D(\psi)\right] P(\psi, t) .
$$

Here, $v(\psi) \equiv D^{(1)}(\psi)$ is the drift coefficient and $D(\psi) \equiv D^{(2)}(\psi)$ is the diffusion coefficient which are directly related to the short-scale evolution of the first two moments of $\psi$ via Eq. (3).

This, in turn, implies that the Fokker-Planck equation components (the drift and the diffusion coefficient) can be fully reconstructed from short-scale simulations. For our coarsegrained dynamics, we initialize the system consistently with some value of the coarse variable $\psi_{0}$ (we call this procedure of constructing microscopic initial conditions consistent with the prescribed coarse variables as "lifting" 14 ). Then we perform a short-scale simulation and estimate the derivatives of the average and the standard deviation of the coarse variable,

$$
v\left(\psi_{0}, t\right)=\frac{\partial\left\langle\psi\left(t, \psi_{0}\right)\right\rangle}{\partial t}, \quad D\left(\psi_{0}, t\right)=\frac{1}{2} \frac{\partial \sigma^{2}\left(t, \psi_{0}\right)}{\partial t}
$$

Here, $\psi\left(t ; \psi_{0}\right)$ is a trajectory of the system that starts from $\psi=\psi_{0}$ at time $t=0$, angular brackets denote averaging over different realizations of this trajectory, and $\sigma^{2}\left(t ; \psi_{0}\right)$ is the variance of $\psi\left(t ; \psi_{0}\right)$. Hence, we can reconstruct a global PDE from short-scale, appropriately initialized local simulations. In practice (in this paper) the derivatives contained in 
expressions (5) are computed by fitting a straight line to $\langle\psi\rangle(t)$ and $\sigma^{2}(t)$. This procedure is discussed in more detail in Section IV clearly, better fitting techniques (e.g. maximum likelihood estimation) can be used. Once the Fokker-Planck equation is reconstructed, one can calculate several global characteristics of the system, such as the effective free energy $G(\psi)$ and the rates of transitions between different metastable states of the system. This effective free energy can be obtained from the equilibrium probability distribution $P_{\text {eq }}(\psi)$ which, in turn, is a solution of the steady-state Fokker-Planck equation

$$
\left[-v(\psi)+\frac{\partial}{\partial \psi} D(\psi)\right] P_{\mathrm{eq}}(\psi, t)=0 .
$$

By substituting the ansatz $P_{\text {eq }}(\psi) \propto \exp \left(-G(\psi) / k_{B} T\right)$ into the equation (6) , we obtain

$$
G(\psi)=-k_{B} T \int \frac{v\left(\psi^{\prime}\right)}{D\left(\psi^{\prime}\right)} d \psi^{\prime}+k_{B} T \ln D(\psi)+\text { const. }
$$

Here, $k_{B}$ is the Boltzmann factor and $T$ is the temperature of the system. Note that, since the free energy is defined up to an additive constant, one can multiply $D(\psi)$ in the logarithmic term by an arbitrary constant in order to preserve consistent units. The second term of Eq. (77) is significant if the noise is multiplicative. Since this is a subtle point that can be overlooked if one uses other (equivalent) descriptions of the stochastic process, we here discuss it in more detail.

Let us first discuss the connection between the Fokker-Planck equation and the corresponding Langevin equation descriptions. This point would become important if we tried to fit simulation data to a coarse-grained Langevin description -rather than a coarse-grained FP description.

A Langevin equation that corresponds to the Fokker-Planck equation (4) is as follows:

$$
\dot{\psi}=\frac{1}{\gamma(\psi)}\left(f_{0}(\psi)+\Gamma(t)\right)
$$

Here, $\gamma(\psi)$ is the friction coefficient, $f_{0}(\psi)$ is the deterministic force, and $\Gamma(t)$ is the stochastic force. The latter is a Gaussian stochastic process with zero mean and with variance related to the damping coefficient $\gamma$ by the fluctuation-dissipation theorem:

$$
\langle\Gamma(t) \Gamma(t+\tau)\rangle=2 \gamma k_{B} T \delta(\tau)
$$

The relationship between $f_{0}(\psi)$ and $\gamma(\psi)$ of the Langevin equation and the drift and diffusion coefficients $v(\psi)$ and $D(\psi)$ of the Fokker-Planck equation depends on the interpretation of the white noise in the Langevin equation (8) as discussed in standard references 
(see e.g. Refs. 13,15). If one uses Itô interpretation, then

$$
\begin{aligned}
v(\psi) & =f_{0}(\psi) / \gamma(\psi), \\
D(\psi) & =k_{B} T / \gamma(\psi),
\end{aligned}
$$

and, if one use the Stratonovich interpretation, then

$$
\begin{aligned}
v(\psi) & =f_{0}(\psi) / \gamma(\psi)-\frac{\gamma^{\prime}(\psi)}{2 \gamma^{2}(\psi)} k_{B} T, \\
D(\psi) & =k_{B} T / \gamma(\psi) .
\end{aligned}
$$

Both of these interpretations are identical in the case of additive noise (i.e. when $\gamma$ is a constant independ on $\psi$ ). In the case of multiplicative noise (i.e. when $\gamma$ is depends on $\psi$ ), the situation becomes somewhat more complicated and, in particular, the force $f_{0}(\psi)$ is not just a gradient of the free energy $G(\psi)$ for both Itô and Stratonovich interpretation. This can be confirmed by direct substitution and is discussed in detail in Ref. 16. In particular, in the case of Itô interpretation,

$$
f_{0}(\psi)=-G_{0}^{\prime}(\psi)
$$

where

$$
G_{0}(\psi)=-k_{B} T \int \frac{v(\psi)}{D(\psi)} d \psi+\text { const. }
$$

It is clear that the expressions (71) and (15) are identical up to an additive constant only if the diffusion coefficient $D$ (and hence the damping coefficient $\gamma$ ) is constant. Therefore, we compute the effective free energy using Eq. (77).

Despite the fact that Eq. (15) is an incorrect expression for the free energy, the quantity $G_{0}(\psi)$ finds its use in calculations of transition rates. In fact, the mean residence time in a free energy well can be written as 15

$$
\tau=\int_{\psi_{0}}^{\psi} d y e^{G_{0}(y) / k_{B} T} \int_{y}^{\infty} d z e^{-G_{0}(z) / k_{B} T} D(z),
$$

where $\psi$ is a point inside the well, $\psi_{0}$ is the boundary of the well. This expression is used in section IV to compute the micelle (computational) disintegration rate. If the free energy barrier is sufficiently high, then transitions such as micelle formation and disintegration, can be described by first order kinetics and the transition rate $k$ is the inverse of the mean residence time $\tau$.

Earlier work on such a kinetic approach ${ }^{7}$ has used the Langevin equation description of the stochastic process. The information about the system dynamics was obtained from the 
time derivatives of $\langle\psi\rangle(t)$ and $\sigma^{2}(t)$ which, in turn, were obtained by fitting $\langle\psi\rangle(t)$ and $\sigma^{2}(t)$ to a straight line, just like for the FP equation description, see Eqs (5). Therefore, the fitting procedure for the Langevin equation model is the same as that for the FP equation model. However, if the diffusion coefficient is not constant, the interpretation of the fitting results for the Langevin equation can lead to ambiguities since, in this case, one would have to specify an interpretation of the white noise (Itô or Stratonovich). We will bypass here the details of the estimation (fitting) of the data to a Langevin description that arise from the interpretation dilemma, and use the Fokker-Planck description of the stochastic process which directly relates the fitted drift and diffusion coefficients to the statistical properties of the process.

Another popular description of a stochastic process is the Smoluchowski equation

$$
\frac{\partial P(\psi, t)}{\partial t}=\frac{\partial}{\partial \psi} D(\psi)\left[-\frac{f(\psi)}{k_{B} T}+\frac{\partial}{\partial \psi}\right] P(\psi, t),
$$

which was originally derived from a Fokker-Planck equation for an inertial Brownian particle in the limit of negligible inertia ${ }^{13}$. The advantage of the Smoluchowski equation is that $f(\psi)$

is the "true" effective force, i.e. $f(\psi)=-G^{\prime}(\psi)$. However, $f(\psi)$ of the Smoluchowski equation, in general, is not proportional to the drift coefficient $v(\psi)$ discussed earlier. In fact, some straightforward algebra shows that

$$
-G^{\prime}(\psi)=f(\psi)=\frac{v(\psi)-D^{\prime}(\psi)}{D(\psi)} k_{B} T,
$$

which is consistent with Eq. (77). Hence, the correction due to the position-dependent diffusion coefficient (the second term in Eq. (77) is present also in the Smoluchowski equation.

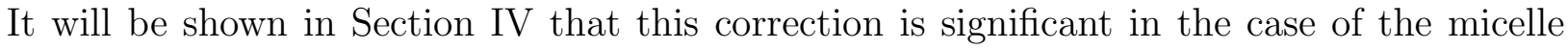
formation, where the diffusion coefficient is significantly inhomogeneous.

\section{MODEL AND EQUILIBRIUM SIMULATION DETAILS.}

We study the micellization process using the lattice model for surfactant systems originally proposed by Larson $\stackrel{8.9}{2}$. Panagiotopoulos and coworkers $\stackrel{17.18}{\underline{10}}$ have performed extensive grand canonical Monte Carlo (GCMC) simulations of this model in order to study micellization and phase transitions. Despite its simplicity, this model yields predictions that are in good qualitative agreement with experimental data. 
In this model, an amphiphile molecule is represented as a chain of beads and a solvent molecule is represented as a single bead. The beads occupy sites on a cubic lattice and the connected beads of an amphiphile molecule are restricted to be in nearest-neighbor sites with bonds along the vectors $(0,0,1),(0,1,1),(1,1,1)$ and their reflections along the principal axis, resulting in a coordination number of 26 . There are two types of beads: hydrophobic tail $(\mathrm{T})$ and hydrophilic head $(\mathrm{H})$ and the solvent is modeled by head beads.

The hydrophobic interaction is modeled by attractive interaction between tail beads. Each bead interacts only with the 26 nearest neighbors and the total energy of the system is the sum of pairwise interactions between beads. The tail-tail interaction energy $\epsilon_{T T}$ is -2 and the tail-head and head-head interaction energies $\epsilon_{T H}$ and $\epsilon_{H H}$ are zero, following Ref. 18 . It is furthermore assumed that all sites that are not occupied by the amphiphile beads are occupied by the solvent. This assumption implies that there is no need to explicitly include solvent into the MC moves.

In most calculations presented in this paper, the following mix of MC moves is used: $50 \%$ amphiphile transfers (i.e. addition or removal), 49.5\% amphiphile partial regrowth moves, and $0.5 \%$ cluster moves. In Section $\mathrm{VC}$, we perform simulations with several different mixes of MC moves in order to investigate the effects of different "dynamics" on the kinetic approach results. Since in this paper we apply a dynamic approach to equilibrium MC simulations, in order to simplify the notation, we refer to the number of $\mathrm{MC}$ move as the "time". Let us emphasize once again that it is the $M C$ computational dynamics that we attemp to -in some sense- accelerate, and not physical dynamics; when the base simulation is an MD one (as in Ref. 7) then our approach would attempt to accelerate physical dynamics.

The simulations are performed for an amphiphile chain $\mathrm{H}_{4} \mathrm{~T}_{4}$ which consists of 4 head beads and 4 tail beads. The simulations are performed at temperature $k_{B} T=7.0$ and chemical potential $\mu=-47.40$ in a cubic box with a side length of 40 sites, assuming periodic boundary conditions. This box size is sufficient to prevent spurious effects of periodicity, since the typical diameter of a micelle is significantly smaller than half the size of the box side.

We perform a reference long-time simulation of the system in order to compute the free energy and the rates of creation and destruction of micelles. In this simulation, we consider 500 realizations of the system and compute the above quantities using data saved from 600 million MC steps after equilibration. The free energy curve $G$ is parameterized by the 
cluster size $\psi$ and is computed from the histogram of the cluster sizes. A cluster is defined as an aggregate of amphiphile molecules such that each molecule in a cluster has at least one tail bead which occupies a neighboring site with a tail bead from another amphiphile of the cluster. In other words, each cluster molecule interacts through hydrophobic attraction with at least one other cluster molecule. The cluster size $\psi$ is defined as a number of amphiphiles in this cluster. The free energy $G_{\text {eq }}(\psi)$ obtained from these equilibrium calculations is shown by the solid line in Fig. 19. $G_{\text {eq }}(\psi)$ has two minima: one at $\psi=1$ which corresponds to free amphiphiles and another one at $\psi=69$, which corresponds to micelles. The free energy barrier separating these two states is located at $\psi_{b} \approx 21$.

In the calculations of equilibrium micelle formation/disintegration rates, a transition between a small cluster and a micelle is said to occur when the cluster size crosses the free energy barrier $\psi_{b}$. For the purposes of the equilibrium calculation, the precise definition of the border between micelles and smaller clusters is unimportant since the transition happens on the much faster timescale than the average lifetime of a micelle. The rate of micelle formation/disintegration or, in order words, of transition from a system containing $i$ micelles to a system containing $i \pm 1$ micelles in a simulation box, is

$$
k_{i \rightarrow i \pm 1}=1 / \tau_{i->i \pm 1}
$$

where $\tau_{i->i \pm 1}$ is the average time between the transitions. Eq. (19) follows from the first order kinetics approximation, which is justified when the time between micelle birth/death has an exponential distribution. This assumption holds if the free energy barrier is sufficiently high (which is true in the current case) and, moreover, we have checked this assumption by direct calculation of the transition time distribution. We expect that the first order kinetics assumption will break down in denser systems, where the micelle coalescence becomes a dominant mechanism for altering size and number density of micelles. However, as discussed in Paper I, the system under consideration $\left(\mathrm{H}_{4} \mathrm{~T}_{4}\right.$ at $k_{B} T=7.0$ and $\left.\mu=-47.40\right)$ has low micelle density with an average of about 1 micelle per $40 \times 40 \times 40$ simulation box.

In the system considered here, there are no long-range energetic interactions between the micelles. However, we observe that, due to entropic interactions, the micelle birth and death rates vary depending on the number of micelles already present in the simulation box. In this work, we focus on transitions $0 \rightarrow 1$ and $1 \rightarrow 0$, i.e. birth and death of micelles in a simulation box that is otherwise filled only with small clusters. An extension to a general 
case of transitions $i \rightarrow i \pm 1$ is straightforward.

\section{DETAILS OF "KINETIC" SIMULATIONS.}

In this section, we describe the details of the implementation of our kinetic approach for the computational micelle formation. As discussed in section II in order to compute the drift and diffusion coefficients, we perform short-time simulations initialized at a prescribed value $\psi_{0}$ of the coarse variable $\psi$. In the case of micelle formation, $\psi$ is the number of amphiphile molecules contained in a micelle (or a nucleus of a micelle). Hence, in order to initialize the simulations, we place a cluster of a prescribed size $\psi_{0}$ into the simulation box.

In order to facilitate this process, we maintain a database of cluster structures. In the simulations reported here, the database is obtained from an equilibrium simulation by saving cluster structures every 100,000 MC steps. As will be shown in section VI, this frequency of the database updates assures that the saved structures are sufficiently different from each other. The database thus obtained contains equilibrium structures of clusters for some temperature $T$ and chemical potential $\mu$. In the current paper, we consider the kinetic approach precisely for these values of $T$ and $\mu$. However, it is very straightforward to generate a new cluster database from an existing one: it is only necessary to equilibrate the available cluster structures at new $T$ and $\mu$; we will estimate the (relatively short) time of this equilibration below.

Thus, the initial conditions for each simulation of the kinetic approach consist of a single cluster of size $\psi_{0}$ picked at random from the database and placed into an empty simulation box. The values of $\psi_{0}$ range from 1 to 90 and, for each $\psi_{0}$, 3000 to 10,000 MC realizations are computed. In addition to the micelle (or a nucleus) which is explicitly placed into the system, the system always contains some "soup" of single amphiphiles, dimers, and other small clusters. Since we do not put these small clusters into the system explicitly, we let it equilibrate before computing statistics of the nucleus evolution. Equilibration here means reaching a quasi-steady-state distribution of small clusters. In order to obtain the small clusters equilibration time, we compute the evolution of average small cluster size and the first two moments of the distribution of the number of molecules contained in small clusters

and conclude that these quantities reach their steady-state values within just 20,000 MC steps. 
We hence use the equilibration time of 20,000 MC steps or more in our simulations. In the non-equilibrium results reported below, time $=0$ corresponds to the time at which the small clusters have equilibrated. Since the nucleus size can change a little during the equilibration time (due to addition/removal of amphiphiles to/from the nucleus), $\psi_{0}$ refers to the size of the cluster after the small cluster equilibration is complete. We will also discuss below the option of small cluster equilibration constrained on the cluster size (in the spirit of umbrella sampling).

The nucleus size $\psi(t)$ is measured with some prescribed frequency $\Delta t$ and the center of mass of the nucleus is tracked in order to prevent possible confusion between a small "dying" nucleus and an emerging small cluster. We have performed two series of MC simulations:

1. Long simulations: Length of production run $=15 \times 10^{4}$ steps; frequency of output $\Delta t=1000$ steps; equilibration time before production run $=5 \times 10^{4}$ steps.

2. Short simulations: Length of production run $=2 \times 10^{4}$ steps; frequency of output $\Delta t=100$ steps; equilibration time before production run $=2 \times 10^{4}$ steps.

The long simulations have been performed in order to study slower dynamics of (almost) formed micelles inside the free energy well; the short simulations have been performed in order to study faster dynamics near the free energy barrier as well as to explore the dynamics of the additional coarse variables (see sections $\nabla$ and $\nabla \mathrm{I}$ ). We observe that results of both simulations agree for the fast dynamics near the barrier but the shorter simulations fail to provide sufficient information to correctly reconstruct the free energy surface corresponding to slower dynamics near the free energy minimum. We hence report the free energy and the diffusion coefficient obtained from the longer simulations.

From the MC results, we compute $\left\langle\psi\left(t, \psi_{0}\right)\right\rangle$ and $\sigma^{2}\left(t, \psi_{0}\right)$ and obtain the time derivatives of these quantities by fitting a straight line to them. An example of obtained $\left\langle\psi\left(t, \psi_{0}\right)\right\rangle$ and $\sigma^{2}\left(t, \psi_{0}\right)$ together with the fitted lines is shown in Fig. 2. The fitting is performed for $t \in\left[t_{1}, t_{2}\right]$, where $t_{1}$ and $t_{2}$ are cut-off times, whose choice is motivated by the following considerations. The evolution for $t<t_{1}$ is neglected, since it corresponds to "healing" the details of our particular initialization as we approach the one-dimensional manifold parameterized by $\psi$. The one-dimensional coarse-grained description for $\langle\psi\rangle(t)$ becomes a valid approximation after some initial time has elapsed, i.e. beyond $t_{1}$. The cut-off time $t_{1}$ was chosen by a visual inspection of the plots and its precise choice does not influence 
the results. The relation of the multi-dimensional dynamics to $t_{1}$ will be discussed in more detail in section VI.

The upper cut-off time $t_{2}$, corresponding to the evolution of the initial $\delta$-function density becoming non-Gaussian, can be justified as follows. If the initial cluster size $\psi_{0}$ is sufficiently small, then a significant fraction of MC realizations will result in a complete disintegration of the nucleus into a collection of unconnected single amphiphiles. This process is illustrated in Fig. 3. which shows an evolution of the distribution of the cluster size. Initially, this is a $\delta$-function distribution. At some intermediate time, this is still well approximated by a Gaussian distribution; we have not yet started to sample the nonlinearities of the effective free energy away from the nominal initial point. At some later time, when a significant fraction of clusters has disintegrated, the distribution starts becoming bimodal. This bimodality of the distribution is echoed in a nonlinear (in time) behavior of both $\langle\psi\rangle(t)$ and $\sigma^{2}(t)$. We hence choose the upper cut-off time $t_{2}$ as the time at which the height of the second mode is $5 \%$ the height of the Gaussian mode.

We observe that the introduction of the upper cut-off time $t_{2}$ is necessary only for relatively small clusters $\left(\psi_{0} \leq 30\right)$. For larger clusters, $t_{2}$ is much larger than the simulation time because disintegration of a micelle into small clusters is an extremely slow process, and the simulation does not leave the neighborhood of the bottom of the micelle well.

\section{RESULTS}

The effective free energy $G(\psi)$ obtained from the kinetic approach is compared to the free energy $G_{\text {eq }}(\psi)$ obtained from the full-scale equilibrium simulations in Fig. 1a. We observe good agreement between the two estimates of the free energy for the values of $\psi$ located on the right of the free energy barrier. The discrepancy between $G(\psi)$ and $G_{\text {eq }}(\psi)$ becomes significant of the left of the barrier. As will be discussed in Section VI, we believe that this discrepancy is due to the fact that the dynamics for these small $\psi$ is effectively multi-dimensional, i.e. the timescale of the approach to the one-dimensional manifold is comparable to the timescale of motion along that manifold.

The effective diffusion coefficient $D(\psi)$, shown in Fig. 1b, exhibits strong position dependence near the free energy barrier. This suggests importance of the correction to the free energy due to multiplicative noise (see the second term in Eq. (17)). Indeed, in Fig. 17 we 
show for comparison the free energy $G_{0}(\psi)$ obtained from expression (15), which neglects the multiplicative nature of the noise. It is clear that the discrepancy between $G_{0}(\psi)$ and $G_{\text {eq }}(\psi)$ is significant in the barrier region, precisely in the region of strong position dependence of $D(\psi)$.

Drift and diffusion coefficients $v(\psi)$ and $D(\psi)$, obtained from the kinetic approach calculations, can be used to obtain the computational disintegration rates of micelles. From Eq. (16), we compute the micelle disintegration rate to be $k=5.58 \times 10^{-9}$. This compares reasonably well with the micelle disintegration rate of $k_{1 \rightarrow 0}=7.70 \times 10^{-9}$ obtained from the full-scale MC simulations (see Section [II). The discrepancy is partly due to the discrepancy in free energies on the left of the saddle point (see Fig. 17). In fact, if we compute the disintegration rate using the free energy obtained from the equilibrium simulation and the diffusion coefficient obtained from the kinetic approach calculations, we obtain the rate $k=6.58 \times 10^{-9}$, in a better agreement with the equilibrium result.

Calculation of the micelle formation rate is somewhat more complicated because in this case one needs to examine dynamics of very small nuclei, which cannot be described by our one-dimensional Fokker-Planck equation parametrized by micelle size. In fact, a small nucleus is indistinguishable from other small clusters in the simulation box. A possible solution is to match the flux $j_{+}(\psi)$ of growing cluster sizes with the flux $j_{-}(\psi)$ of the decaying cluster sizes $\psi$. The flux $j_{+}(\psi)$ of nuclei emerging from the "soup" of small clusters can be calculated directly using short-scale simulations with initial conditions being an empty box. The flux $j_{-}(\psi)$ of disintegrating clusters can be calculated from the Fokker-Planck equation. In order to match these fluxes, it is required to have a reliable FP equation description of the cluster size evolution in the range of $\psi$ where the matching is expected to take place. However, currently we observe a discrepancy between the equilibrium simulations and the kinetically fitted single coarse variable Fokker-Planck equation on the left of the free energy barrier $\psi_{b}$, which is evidenced, e.g. by different slopes of $G_{\text {eq }}(\psi)$ and $G(\psi)$ on the left of $\psi_{b}$ (see Fig. 19). Since the matching should be performed for $\psi<\psi_{b}$, we cannot currently estimate micelle formation rates using the the kinetic approach using the micelle size as a "coarse variable". However, the effective Fokker-Planck equation description on the left of $\psi_{b}$ can be improved if one goes beyond the one-dimensional coarse variable model, as discussed in section VI 


\section{A. Validity of the Fokker-Planck equation assumptions.}

In this subsection, we discuss several assumptions behind the effective FP equation dynamics and demonstrate computationally that these assumptions hold in the case of our GCMC simulations of micelle formation. One of the assumptions implicit in the FP model is that the cluster size $\psi$ changes gradually, i.e. removal (addition) of single amphiphiles (or, possibly di- and tri-mers) from (to) the nucleus is far more probable than spontaneous break up of a nucleus into several clusters of comparable size (spontaneous assembly of clusters into a nucleus). In order to check this assumption, we compute the probability $P(\Delta \psi ; \psi)$ of removal (addition) of a cluster of size $\Delta \psi$ from (to) the nucleus of size $\psi$. We observe that, for all nuclei sizes, removal/addition of a single amphiphile has a probability greater than 0.9 and the probabilities of removal/addition of larger clusters decrease monotonically with the cluster size. In Fig. 4, we show the probability $P(\Delta \psi ; \psi)$ for the nucleus size $\psi=10$. Such probability distributions are almost identical for all nucleus sizes $\psi \geq 10$ and hence, the assumption of the gradual change of the size of the nucleus is valid.

Another assumption of the FP equation is that the process is Markovian. This assumption is equivalent to the assumption (9) of the zero-correlation time of the stochastic force in the Langevin equation (8). From the Langevin equation, it follows that the correlation time of noise coincides with that of $d \hat{\psi}(t) / d t$, where

$$
\hat{\psi}(t)=\psi(t)-\langle\psi(t)\rangle
$$

is the fluctuation of $\psi(t)$. Therefore, in order to estimate the correlation time of $F(t)$, we compute the autocorrelation function $d \hat{\psi}(t) / d t$. The time derivative of $\hat{\psi}(t)$ is estimated using the forward differences,

$$
\left.\frac{\hat{\psi}(t)}{d t}\right|_{t=t_{i}} \approx \frac{\hat{\psi}\left(t_{i+1}\right)-\hat{\psi}\left(t_{i}\right)}{\Delta t}
$$

where $\Delta t$ is the frequency of output in our simulations. In the calculations of the autocorrelation functions we have used results of the shorter MC simulation with more frequent output (see Section [V] and hence $\Delta t=100 \mathrm{MC}$ steps. A normalized autocorrelation function of $\hat{\psi}(t)$ (and, hence, of $F(t)$ ) for the initial nucleus size $\psi_{0}=12$ is shown in Fig. 5 and is typical for all $\psi_{0} \geq 10$. It is clear that approximating the effective stochastic practically by white noise is a good assumption. Hence, the evolution of the nucleus size can be modelled by the effective Fokker-Planck equation. 


\section{B. Quality of database}

Another important question that needs to be addressed is whether the cluster database has a sufficient number of cluster structures in order to provide statistically accurate initial conditions for the kinetic approach simulations. This question is especially pertinent near the free energy barrier. Recall that the cluster database is obtained from the equilibrium run and the clusters are saved every 100,000 MC steps. Since the probability to observe a cluster near the barrier is very low, there is a big difference in the number of the available cluster structures at the barrier and in the free energy well. The database used in most of our calculations was obtained from 500 realizations of 5 million MC steps and, although there are hundreds of entries for (almost) equilibrium micelles in the free energy well, there is as little as 3 database entries for some cluster sizes near the barrier. In order to check if this small number of initial configurations introduces a bias into the kinetic approach simulations, we have added more structures to the database by running equilibrium MC simulations for additional 45 million MC steps. In this larger database, the smallest number

of database entries is 118. We have repeated the calculations with this enlarged database and obtained the same $G(\psi)$ and $\gamma(\psi)$ as we did with the smaller database. Hence, the kinetic approach calculations are accurate even for small number of database entries. This happens because, even if initially we place the same nucleus into several copies of the simulation box, during the equilibration time these nuclei will evolve into statistically different structures. The timescales of change of the cluster structures, as well as biasing the equilibration by constraining the nucleus size will be discussed in Section VI.

\section{Role of different dynamic rules.}

Since MC simulations do not reflect the real physical dynamics and the choice of MC moves is somewhat arbitrary, the kinetic properties obtained from MC simulations (such as rates of micelle formation and disintegration) are expected to change as we change the $\mathrm{MC}$ rules. However, if the Fokker-Planck model is valid for the MC "dynamics", the equilibrium properties (such as the free energy), obtained from the kinetic approach should not be affected by the change of the MC rules.

In order to confirm this, we perform MC simulations using 9 different mixes of $\mathrm{MC}$ 
moves, which we call mix $0, \ldots, \operatorname{mix} 8$ (mix 0 corresponds to the simulations reported in the preceding sections). Probabilities of different MC moves in these mixes are listed in Table【. The acceptance/rejection ratios for MC moves in all simulations are observed to be identical.

The simulations are performed near the free energy barrier, with the initial nucleus size $\psi_{0}$ ranging from 10 to 40 . As expected, the "dynamics" is different for different mixes of MC moves. This can be seen e.g. in Fig. [6] which compares evolutions of $\langle\psi\rangle(t)$ and $\sigma^{2}(t)$ computed from the simulations with rules mix 7 and mix 8. However, the free energy curves reconstructed from the short-scale kinetic approach MC simulations agree for different MC "dynamics", as seen in Fig. 7. This agreement becomes somewhat worse on the left of the free energy barrier $\psi_{b}$. The obtained results thus confirm that the Fokker-Planck model provides an adequate description for the "dynamics" of MC simulations of the micelle growth and decay.

\section{MULTI-DIMENSIONAL DYNAMICS.}

In the previous sections, we have discussed Monte Carlo "dynamics" of micelle formation assuming that the system can be accurately modeled by a single coarse variable, namely, the aggregation number $\psi$ of a cluster. The aggregation number provides sufficient information about a micelle at equilibrium. In fact, one can reconstruct the thermodynamic properties of an equilibrium micelle of a given aggregation number using a mean-field theory ${ }^{19.20 .21}$. However, non-uniformities of non-equilibrium clusters might prevent a unique specification of structure and physical properties of these clusters.

In this section, we explore the "evolution" of cluster structures and consider "dynamics" in a two-dimensional $\psi-E$ space, where $E$ is the cluster energy. This variable is a useful probe of the cluster structure because if reflects how tightly the cluster is packed: the smaller the energy, the more hydrophobic groups are in contact. We note that one can choose a different variable (e.g., a radius of gyration) to reflect the cluster structure. However, the specific choice of the second coarse variable is not important: if the coarse-grained cluster dynamics are successfully parameterized by two variables, then all other variables in our simulation become quickly slaved to the chosen two coarse variables.

It is more convenient to consider dynamics of the normalized cluster energy $E / \psi$. Fig. 8 
shows averaged trajectories in the $\psi-(E / \psi)$ phase space. The trajectories are obtained from the short-scale simulations described in the previous sections. For each initial nucleus size $\psi_{0}$, we compute minimum and maximum energies $E_{\min }$ and $E_{\max }$ of the nuclei of this size at time $\mathrm{t}=0$ (i.e., just after equilibration). Then, the range of energies $\left[E_{\min }, E_{\max }\right]$ is divided into 10 equal intervals and the trajectories that begin at the same $\psi_{0}$ and in the same energy interval are averaged. The free energy $G(\psi, E / \psi)$, whose contour plot is also shown in Fig. 8, has been obtained from the full-scale equilibrium simulation, described in section III.

It is clear that the trajectories quickly approach a one-dimensional manifold parameterized by the cluster size $\psi$. The equilibrium micelles correspond to a stable node at $\psi=69$ and the free energy barrier corresponds to a saddle point at $\psi=21$. The two-dimensional dynamics provides a clear explanation for the nonlinear behavior of $\langle\psi\rangle$ and $\sigma^{2}(t)$ for $t<t_{1}$ (see Fig. 2 and discussion in section [V]). For clarity, in Fig. 9] we plot several typical trajectories from the complete phase portrait of Fig. 8 and indicate the part of the trajectories with $t<t_{1}$ by thin lines. From these plots, it is obvious that $t_{1}$ corresponds to the time it takes the trajectory to approach the one-dimensional manifold and hence, for $t<t_{1}$, a one-dimensional projection $\psi(t)$ of the trajectory is a nonlinear function of "time" $t$. We emphasize that $t_{1}$ is not the equilibration time since the clusters are already equilibrated prior to computing the averages. However, due to statistical fluctuations in an equilibrated system, there is always a significant fraction of clusters away from the one-dimensional manifold. As Figs. 8 and 9 show, these clusters, on average, will approach this manifold.

Next, we compare the timescales of motions towards the one-dimensional manifold and motion along the manifold near the critical points (saddle point and minimum) of the free energy. Near these points, the averaged dynamics can be approximately described by a linear homogeneous system of differential equations,

$$
\frac{d}{d t}\left(\begin{array}{c}
\langle\psi\rangle \\
\langle E\rangle
\end{array}\right)=A\left(\begin{array}{c}
\langle\psi\rangle \\
\langle E\rangle
\end{array}\right),
$$

where $A$ is a $2 \times 2$ constant matrix, whose eigenvalues $\lambda_{1}$ and $\lambda_{2}$ provide information on the timescale of motion towards and along the manifold. In order to obtain the eigenvalues $\lambda_{1}$ and $\lambda_{2}$, we compute functions

$$
F_{1}\left(\psi_{0}, E_{0} ; t\right)=\langle\psi\rangle(t)-\psi_{0} \quad \text { and } \quad F_{2}\left(\psi_{0}, E_{0} ; t\right)=\langle E\rangle(t)-E_{0},
$$


where $\psi_{0}$ and $E_{0}$ are the values of $\langle\psi\rangle(\mathrm{t})$ and $\langle E\rangle(\mathrm{t})$ at time $t=0$. The eigenvalues $\mu_{1,2}(t)$ of the Jacobian $J(t)$ of the functions $F_{1}\left(\psi_{0}, E_{0} ; t\right)$ and $F_{2}\left(\psi_{0}, E_{0} ; t\right)$ are the multipliers of the linear system (22) and are related to the eigenvalues of the matrix $A$ by the following expression:

$$
\mu_{j}(t)=e^{\lambda_{j} t}-1, \quad j=1,2 .
$$

The Jacobian $J(t)$ is obtained from the least squares fit of $F_{1}\left(\psi_{0}, E_{0} ; t\right)$ and $F_{2}\left(\psi_{0}, E_{0} ; t\right)$ to linear functions of $\psi_{0}$ and $E_{0}$,

$$
F_{j}\left(\psi_{0}, E_{0} ; t\right) \approx J_{j 1}(t) \psi_{0}+J_{j 2}(t) E_{0}+\text { const }, \quad j=1,2 \text {. }
$$

This fitting is performed using data from the averaged trajectories $(\langle\psi\rangle(t),\langle E\rangle(t))$, which start from points $\psi_{0}$ and $E_{0}$ in some neighborhood of a critical point. In particular, in order to estimate the Jacobian near the saddle point at $\psi_{b}=21$, we use trajectories with initial nucleus size $\psi_{0}=18, \ldots, 23$ and we use trajectories with $\psi_{0}=66, \ldots, 74$ to estimate the Jacobian near the minimum at $\psi_{m}=69$. The eigenvalues of matrix $A$ obtained from the multipliers $\mu_{j}(t)$ using Eq (24) are plotted in Fig. 10, After a brief initial transient, these eigenvalues approach steady-state values. The fast eigenvalue $\lambda_{1}$, shown in Fig. [10a, corresponds to motion towards the manifold and, near the saddle point, $\lambda_{1} \approx-9 \times 10^{-5}$ and near the minimum, $\lambda_{1} \approx-4 \times 10^{-5}$. The slow eigenvalue $\lambda_{2}$, shown in Fig. 10p, correspond to motion along the manifold and, near the saddle point, $\lambda_{1} \approx 5 \times 10^{-6}$ and near the minimum, $\lambda_{1} \approx-1.5 \times 10^{-6}$. An order of magnitude separation of timescales appears thus to prevail between the motion towards and that along the one-dimensional slow manifold. We observe that this separation becomes smaller near the saddle point.

The timescale of approaching the one-dimensional manifold provides a useful measure of how often the cluster database should be updated in order for the saved cluster structures to be sufficiently different. It is reasonable to expect that within the time the coarse variables $\psi$ and $E$ have reached the manifold, the corresponding cluster structure is significantly changed. From the eigenvalue analysis presented above, it follows that the timescale of approaching the manifold is on the order of $10^{4} \mathrm{MC}$ steps. Hence, the frequency of the database updates used in our simulations $\left(10^{5}\right.$ steps $)$, ensures that the saved structures are sufficiently different. Moreover, this explains why simulations with a small database produce results almost identical to those of simulations with a larger database (see Section $\mathrm{VB}$ ): the equilibration time of $2 \times 10^{4}$ (for small clusters) and $5 \times 10^{4}$ (for larger clusters) is sufficient 
to significantly alter the nucleus structure and thus to provide good sampling even if one uses a small database. We emphasize that, although in the current work the database was obtained from an equilibrium simulation, it can be also updated on the fly during the kinetic simulation or possibly from an already existing database at some nearby temperature $T$ and chemical potential $\mu$. In this case, estimation of the rate of change of the internal cluster structure is crucial in order to make sure that the database clusters become locally equilibrated.

In order to probe the multi-dimensional dynamics and approach to the one-dimensional manifold, we "prepare" the micelles at the onset of our kinetic simulations by performing preparatory simulations with constrained cluster size. This constraint is implemented by rejecting all MC moves that change the cluster size; approaches like umbrella sampling 22 would also be appropriate in evolving while effectively constraining the cluster size.

We perform two simulations for the cluster size $\psi=13$ starting above and below the one-dimensional manifold. Evolution of cluster radii of gyration $R_{1}, R_{2}, R_{3}$ and energy $E$ are averaged over $500 \mathrm{MC}$ realizations and are shown in Fig. 11. These structural variables approach steady-state values that correspond to the one-dimensional manifold. After the cluster has approached this manifold, we release the constraint and let the cluster size evolve for our kinetic simulation. This evolution of the cluster size is shown in Fig. 9 by thick gray lines (the vertical lines corresponds to the constrained dynamics). After the cluster size is released, the phase trajectory is parallel to the one-dimensional manifold.

The phase trajectories shown in Fig. 9 also provide an explanation for the discrepancy in the free energy $G(\psi)$ on the left of the free energy barrier $\psi_{b}$ (see Fig. 1). Figs. 9a and $9 \mathrm{~b}$ show dynamics on the left and on the right of the barrier, respectively. It is clear that there is a timescale separation between the dynamics of approaching the manifold and motion on the manifold when the trajectory is on the right of the barrier. The timescale separation becomes significantly smaller on the left of the barrier. In addition, on the right of the barrier, the trajectories are much better approximated by a singe one-dimensional manifold for $t>t_{1}$. On the left of the barrier, on the other hand, the trajectories do not quite approach a one dimensional manifold parametrized by cluster size and the slopes of the trajectories which start from the same $\psi$ but different $E$ remain different until complete disintegration of clusters takes place. Hence, in order to correctly reconstruct the free energy for these small cluster sizes, one has to perform an analysis of the two-dimensional dynamics. 
It is interesting to notice that the slope of the one-dimensional effective slow manifold seems to get steeper and steeper as we go towards smaller cluster sizes. To deal with this, we augmented the dimension of the manifold, and added one more coarse observable to parametrize this "fatter" manifold. It is conceivable that one might still be able to get a good one-dimensional coarse description of the dynamics - but at small cluster sizes one would need a different reaction coordinate than the cluster size; one might still have a graph of a function above this new variable, and not need an overall fatter two-dimensional description. Using different order parameters at different areas of phase space, and appropriately patching them together, is a vital area of research in data compression - and we are currently testing this possibility.

\section{DISCUSSION}

We have demonstrated that the Monte Carlo "dynamics" of micelle formation for the Larson model can be successfully described by an effective Fokker-Planck equation, and that the drift and diffusion coefficients of this equation can be obtained from short-scale, appropriately initialized "kinetic" simulations. Due to separation of timescales between the aggregation number $\psi$ of a micelle nucleus and the coarse variables reflecting the nucleus structure (such as the nucleus energy $E$ ), the coarse-grained micelle formation process can be successfully approximated by motion on a one-dimensional manifold parameterized by the coarse variable $\psi$. The separation of timescales becomes weaker for small nucleus sizes and consideration of dynamics in a two-dimensional coarse phase space is necessary for $\psi<\psi_{b}$, where $\psi_{b}=21$ is the location of the free energy barrier.

In addition to the kinetic approach, several other "equation-free" methods are available that can speed up coarse-grained calculations.

\section{A. Coarse Newton method}

The coarse Newton method as well as coarse stability and bifurcation analyses have been described elsewhere ${ }^{23,24.25}$. In the context of the rare events problem, the coarse Newton method can be used to obtain the location of the saddle point. The Newton method was used here to locate zeros of the function $F\left(\psi_{0}\right)$ which is defined as the slope of $\langle\psi\rangle(t, \psi)$. In 
our implementation of the Newton method, the derivative of $F\left(\psi_{0}\right)$ is estimated by fitting a straight line through points $F\left(\psi_{0}-1\right), F\left(\psi_{0}\right)$, and $F\left(\psi_{0}+1\right)$. The results of the iterations of the Newton method initialized at different values of the coarse variable $\psi$ are shown in Fig. 12. Depending on the initial condition, the iterations converge either to the saddle point or to the minimum. It is well known that Newton convergence requires a good initial guess. We did, accordingly, observe that not all initial conditions converge to a stationary point - namely, for some points between $\psi=30$ and 43, the first iteration "shoots" outside of the domain for which the function $F(\psi)$ is defined. This is because $F(\psi)$ is very "flat" for these values of $\psi$ (see the inset in Fig. 12).

The function $F(\psi)$ used in our Newton method can be identified with the driving force $f_{0}(\psi)$ if the Langevin equation (10). The transition states (as well as the free energy minima) correspond to the zeros of the derivative of the free energy $G^{\prime}(\psi)$. In the current implementation of the coarse Newton method, one computes the values of the coarse variable $\psi$ which correspond to the zeros of the slopes of $\langle\psi\rangle\left(t, \psi_{0}\right)$, i.e. the zeros of the drift coefficient. However, as we have seen in Section [II the zero of the drift coefficient does not have to coincide with the zero of the gradient of the free energy (and it is the latter that we are after). In fact, from equation (17) it follows that

$$
G^{\prime}(\psi)=0 \quad \text { if and only if } \quad v(\psi)=D^{\prime}(\psi)
$$

Hence, the zeros of $v(\psi)$ and $G^{\prime}(\psi)$ coincide only if the diffusion coefficient is positionindependent (which is not true in the considered case). However, the results presented in Figs. 1] and 12 indicate that the main correction due to the position dependence of the diffusivity is to the height in the free energy barrier and not the location of the saddle point. Hence, we consider the results of this Newton computation representative of the transition state; implementing a coarse Newton computation with the correction due to the state-dependent noise is straightforward.

\section{B. Coarse reverse integration}

This method has been originally developed for MD simulations in Ref. 7 (see also Ref. 26); after estimating the right-hand-side of an effective Langevin equation, one can effectively reverse the time in a projective coarse Euler step and hence integrate the coarse description 
backwards in time. In coarsely one-dimensional systems the reverse integration converges to a top of the free energy barrier (in contrast to the forward integration which converges to a free energy minimum). In systems described by more than one macroscopic observables (reaction coordinates), coarse reverse integration can be linked with techniques for the construction of stable manifolds of dynamical systems ${ }^{27}$, to efficiently build higher dimensional effective free energy surfaces.

Coarse backward integration can be readily applied to the current system. We perform two series of reverse integration, one starting from the right and the other starting from the left of the free energy barrier and observe that the system indeed converges to the transition state.

\section{Reverse integration starting from the stable micelle}

Results of integration starting from the right of the barrier are shown in Fig. 13] and b. Simulations shown in Fig. [13 start from $\psi_{0}=60$; the duration of inner simulation is $t_{\text {inner }}=2 \times 10^{5} \mathrm{MC}$ steps and the backward projection step is $h=-2 \times 10^{5}$. The solid lines show the short-scale forward simulation results and the dashed lines are the reverse projections. The circles show the initial conditions for the short-scale simulations. When the predicted (projected) state $\psi$ is at a noninteger value of the cluster size, given the coarsegrained nature of the computation, we use an appropriately weighted ensemble of initial cluster sizes bracketing the desired noninteger value.

As $t \rightarrow-\infty$, we observe oscillations in the simulation shown in Fig. 13]. These oscillations are due to the large projection steps: the integrator keeps "overshooting" the free energy barrier. The oscillations can be removed by the reduction in the projective stepsize. This is confirmed by our simulations with a smaller stepsize, shown in Fig 13]b. This simulation is performed starting from $\psi_{0}=31$; duration of the inner simulation is $t_{\text {inner }}=2 \times 10^{4}$

steps and the coarse projection step is $h=-5 \times 10^{4}$. The simulation converges to the location of the free energy barrier. 


\section{Integration starting from the "soup" of small clusters}

Results of the backward integration with initial conditions on the left of the free energy barrier are shown in Fig. 13: and d. Duration of inner forward simulation in these simulations is $t_{\text {inner }}=2 \times 10^{4}$. The integration in Fig. 13r is started from $\psi_{0}=5$ and the timestep for the reverse projection is $h=-2 \times 10^{4}$. The integration approaches a steady state at $\psi=21$, which corresponds to the location of the free energy barrier. As expected, the rate of convergence (measured in terms of the performed iterations) slows down near the barrier. Increasing the projective stepsize to $h=-10^{5}$ approaches the transition state in a smaller number of steps, as shown in Fig. 13d. Adaptive stepsize selection (an established procedure for initial value problems, see e.g. Ref. 28) should in principle be used for best results.

We have therefore demonstrated that the backward timestepper with correctly chosen timestep converges to the location of the free energy barrier.

\section{Summary}

We have successfully applied the coarse kinetic approach to the lattice Monte Carlo simulations of micelle formation. The approach is based on the assumption that the micelle formation "dynamics" can be adequately described by an effective Langevin equation model (and the corresponding Fokker-Planck description) for a few coarse (slow) degrees of freedom, while treating other (fast) degrees of freedom as a thermal noise. The kinetic approach, based on short-scale simulations with judiciously chosen initial conditions, then allows us to adequately reconstruct the free energy surface and the statistical characteristics of the thermal noise.

We have shown that the micelle formation "dynamics" can be parameterized by a single coarse variable, as long as the cluster size is sufficiently large. Investigations of the system dynamics parameterized by an additional coarse variable (e.g. cluster energy), shows existence of a one-dimensional slow manifold, which is quickly approached by the system. This separation of timescales seizes to exist for small cluster sizes. This implies that the early stages of the micelle nucleation can be characterized by "dynamics" on a multidimensional manifold.

We have also briefly demonstrated the application to micelle formation of other "equation- 
free" coarse numerical schemes useful in the context of rare event computations, such as the coarse Newton's method and coarse reverse integration.

\section{Acknowledgments}

This work was partially supported by AFOSR and an NSF/ITR grant. It is a pleasure to acknowledge discussions with Prof. C. W. Gear, Dr. G. Hummer, and Dr. M. Haataja.

* Current address: Department of Chemical Engineering, University of Florida, Gainesville, FL 32611; Electronic address: dkopelevich@che.ufl.edu

$\dagger$ Electronic address: azp@princeton.edu

¥ Corresponding author; Electronic address: yannis@arnold.princeton.edu

1 E. A. Carter, G. Ciccotti, J. T. Hynes, and R. Kapral, Chem. Phys. Lett. 156, 472 (1989).

2 M. R. Sørensen and A. F. Voter, J. Chem. Phys. 112, 9599 (2000).

3 C. Dellago, P. G. Bolhuis, F. S. Csajka, and D. Chandler, J. Chem. Phys. 108, 1964 (1998).

4 P. L. Geissler, C. Dellago, and D. Chandler, J. Phys. Chem. B 103, 3706 (1999).

5 A. Laio and M. Parrinello, Proc. Natl. Acad. Sci. 99, 12562 (2002).

6 W. Cai, M. H. Kalos, M. de Koning, and V. V. Bulatov, Phys. Rev. E 66, 046703 (2002).

7 G. Hummer and I. G. Kevrekidis, J. Chem. Phys. 118, 10762 (2003), can be obtained as physics/0212108 at arXiv.org.

8 R. G. Larson, L. E. Scriven, and H. T. Davis, J. Chem. Phys. 83, 2411 (1985).

9 R. G. Larson, Journal de physique II 6, 1441 (1996).

10 E. A. G. Aniansson and S. N. Wall, J. Phys. Chem. 78, 1024 (1974).

11 J. C. Neu, J. A. Cañizo, and L. L. Bonilla, Phys. Rev. E 66, 061406 (2002).

12 D. I. Kopelevich, A. Z. Panagiotopoulos, and I. G. Kevrekidis, Submitted to J. Chem. Phys. (2004).

13 H. Risken, The Fokker-Planck Equation. Methods of Solution and Applications (Springer-Verlag, Berlin, 1984).

14 I. G. Kevrekidis, C. W. Gear, J. M. Hyman, P. G. Kevrekidis, O. Runborg, and K. Theodoropoulos, Comm. Math. Sciences 1, 715 (2003), original version can be obtained as physics/0209043 
at arXiv.org.

15 G. W. Gardiner, Handbook of Stochastic Methods for Physics, Chemistry, and the Natural Sciences (Springer-Verlag, Berlin, 1983).

16 P. Arnold, Phys. Rev. E 61, 6091 (2000).

17 M. A. Floriano, E. Caponetti, and A. Z. Panagiotopoulos, Langmuir 15, 3143 (1999).

18 A. Z. Panagiotopoulos, M. A. Floriano, and S. K. Kumar, Langmuir 18, 2940 (2002).

19 A. Ben-Shaul, I. Szleifer, and W. M. Gelbart, J. Chem. Phys. 83, 3597 (1985).

20 I. Szleifer, A. Ben-Shaul, and W. M. Gelbart, J. Chem. Phys. 83, 3612 (1985).

21 A. D. Mackie, A. Z. Panagiotopoulos, and I. Szleifer, Langmuir 13, 5022 (1997).

22 G. M. Torrie and J. P. Valleau, Chem. Phys. Letters 28, 578 (1974).

23 C. Theodoropoulos, Y.-H. Qian, and I. G. Kevrekidis, Proc. Natl. Acad. Sci. 97, 9840 (2000).

24 C. W. Gear, I. G. Kevrekidis, and C. Theodoropoulos, Comput. Chem. Eng. 26, 941 (2002).

25 A. G. Makeev, D. Maroudas, and I. G. Kevrekidis, J. Chem. Phys. 116, 10083 (2002).

26 C. W. Gear and I. G. Kevrekidis, SIAM J. Sci. Comput. 24, 1091 (2003), also NEC Technical Report NECI-TR 2001-029, can be obtained as http://www.neci.nj.nec.com/homepages/cwg/projective.pdf.

27 M. E. Johnson, M. S. Jolly, and I. G. Kevrekidis, Numerical Algorithms 14, 125 (1997).

28 W. H. Press, S. A. Teukolsky, W. T. Vetterling, and B. P. Flannery, Numerical Recipes in Fortran 77. The Art of Scientific Computing (Cambridge University Press, Cambridge, 1992), 2nd ed. 


\begin{tabular}{l|l|l|l} 
Mix & Transfer moves & Regrowth moves & Cluster moves \\
\hline 0 & 0.5 & 0.495 & 0.005 \\
1 & 0.5 & 0.4975 & 0.0025 \\
2 & 0.5 & 0.49 & 0.01 \\
3 & 0.4 & 0.595 & 0.05 \\
4 & 0.6 & 0.395 & 0.05 \\
5 & 0.2 & 0.995 & 0.05 \\
6 & 0.8 & 0.195 & 0.05 \\
7 & 0.9 & 0.099 & 0.001 \\
8 & 0.1 & 0.88 & 0.02
\end{tabular}

TABLE I: Probabilities of MC moves in different mixes of rules used in the studies of effects of Monte Carlo "dynamics" on the results of the kinetic approach. 

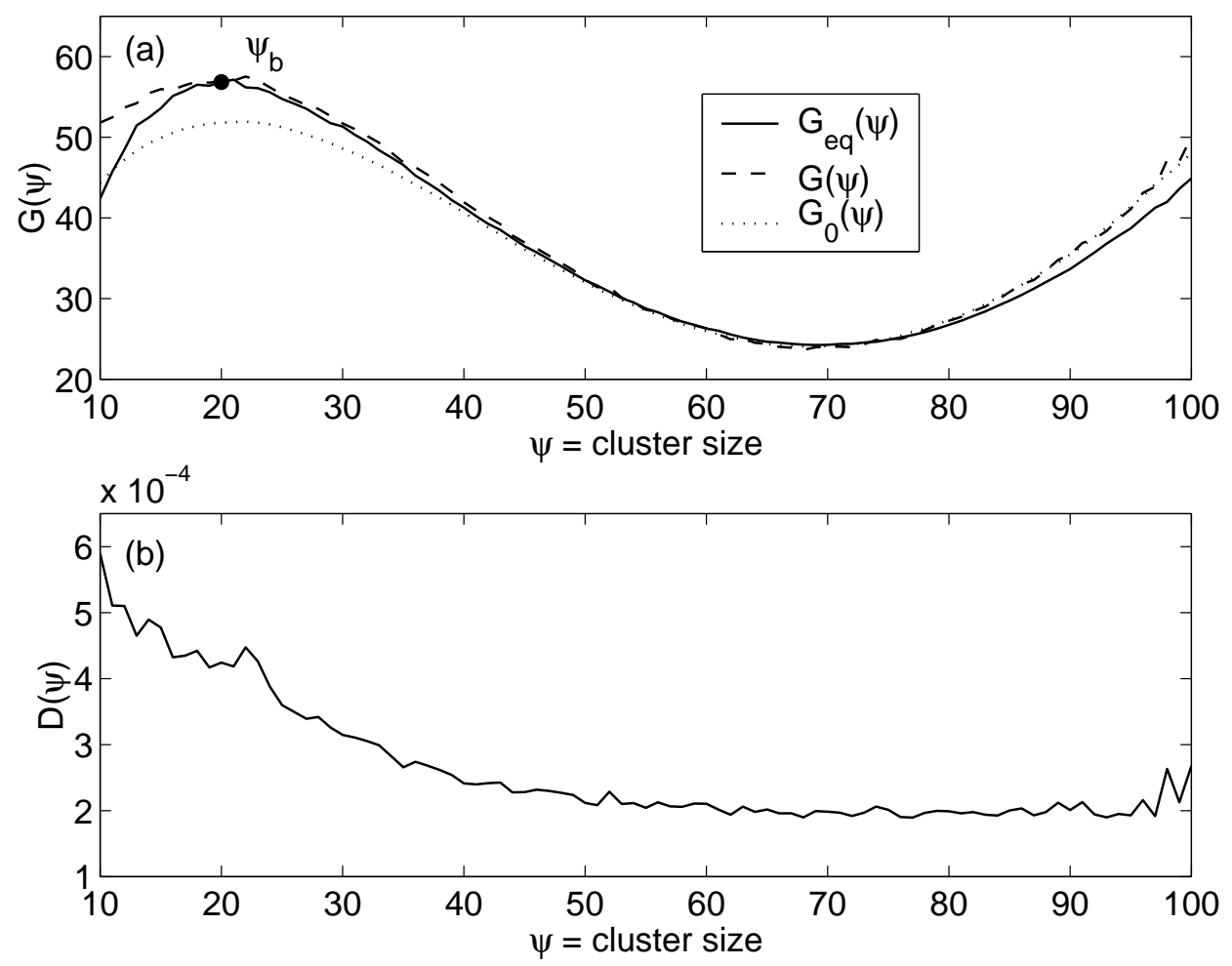

FIG. 1: (a) Free energy $G_{\text {eq }}(\psi)$ obtained from the equilibrium simulations (solid line), free energy $G(\psi)$ calculated using the kinetic approach and Eq. (17) (dashed line), and free energy $G_{0}(\psi)$ obtained from the expression (15) which neglects the spatial dependence of the diffusion coefficient (dotted line); (b) diffusion coefficient $D(\psi)$. 

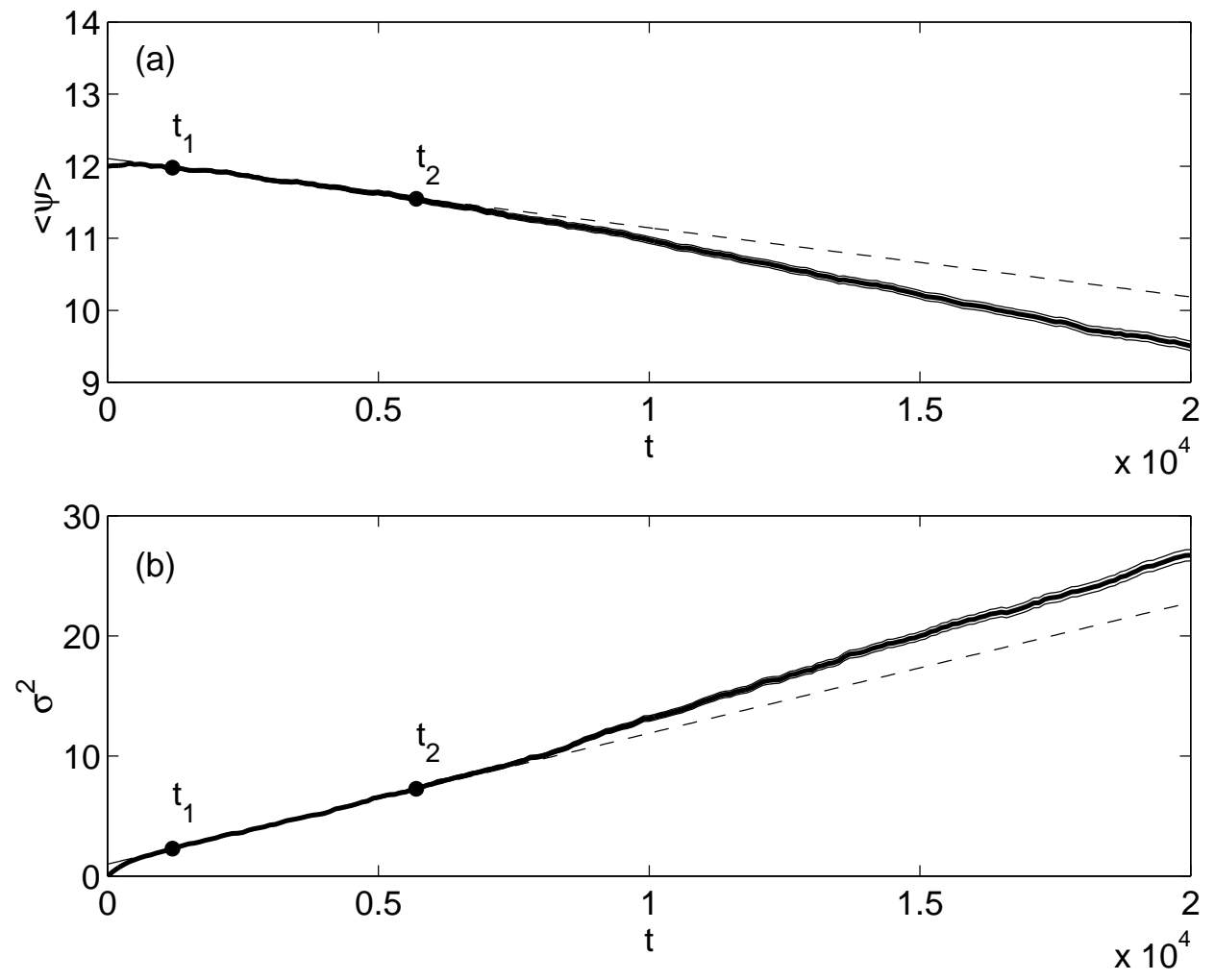

FIG. 2: Evolution of (a) $\left\langle\psi\left(t, \psi_{0}\right)\right\rangle$ and (b) $\sigma^{2}\left(t, \psi_{0}\right)$ for $\psi_{0}=12$. Result of MC simulations are shown by the solid lines with the error estimates bounded by the bands of thin lines; results of the linear least squares fit are shown by the dashed lines and the cut-off times $t_{1}$ and $t_{2}$ are shown by circles. 

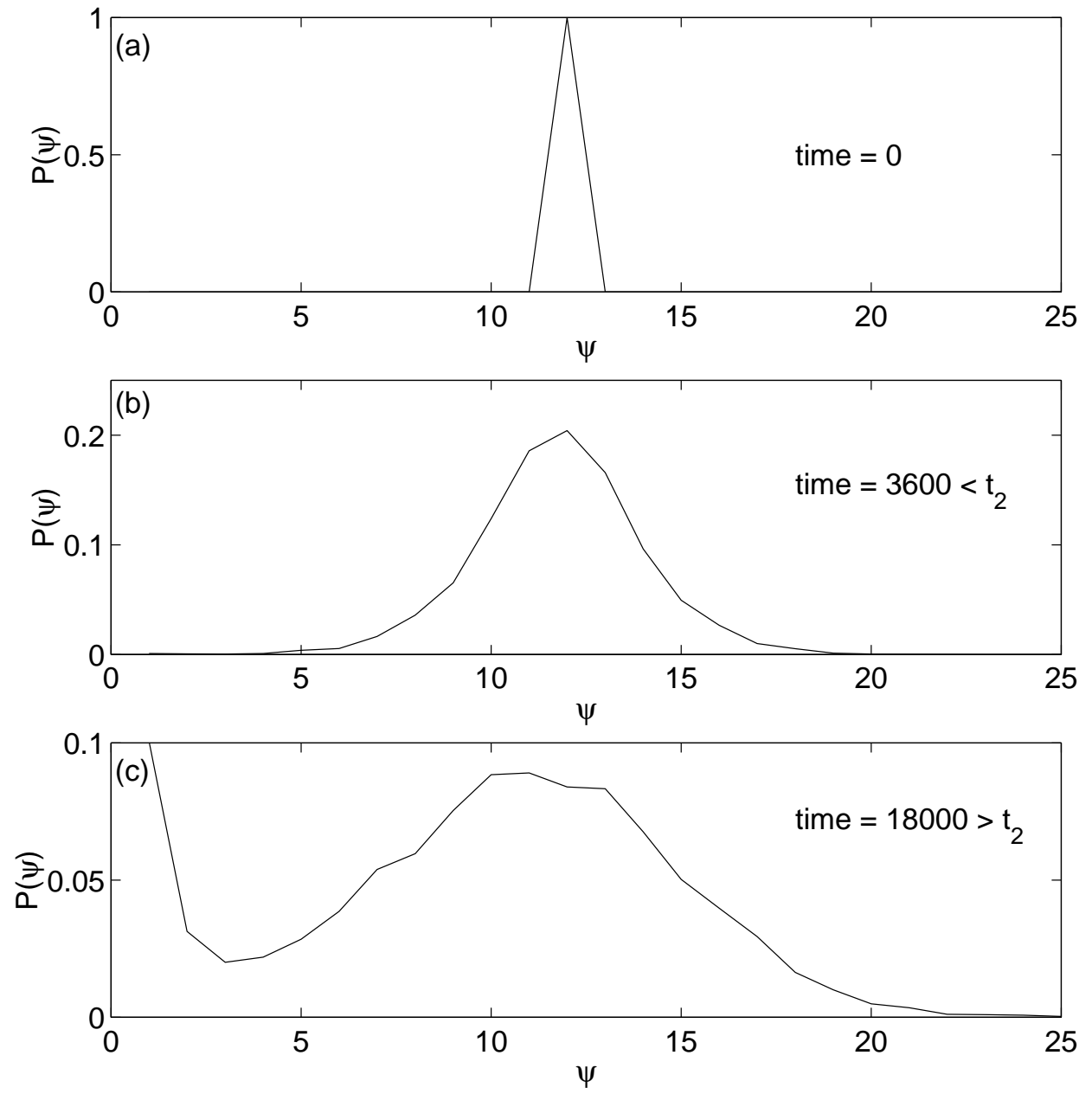

FIG. 3: Evolution of distribution of the cluster size $\psi$. Initial cluster size is $\psi_{0}=12$. (a) $\delta$-function distribution at time $=0$, (b) Gaussian distribution at an intermediate time, and (c) bimodal distribution at a later time, when a significant fraction of nuclei have disintegrated into single amphiphiles, di- and tri-mers (whose dynamics is uncorrelated with $\psi_{0}$ ). 


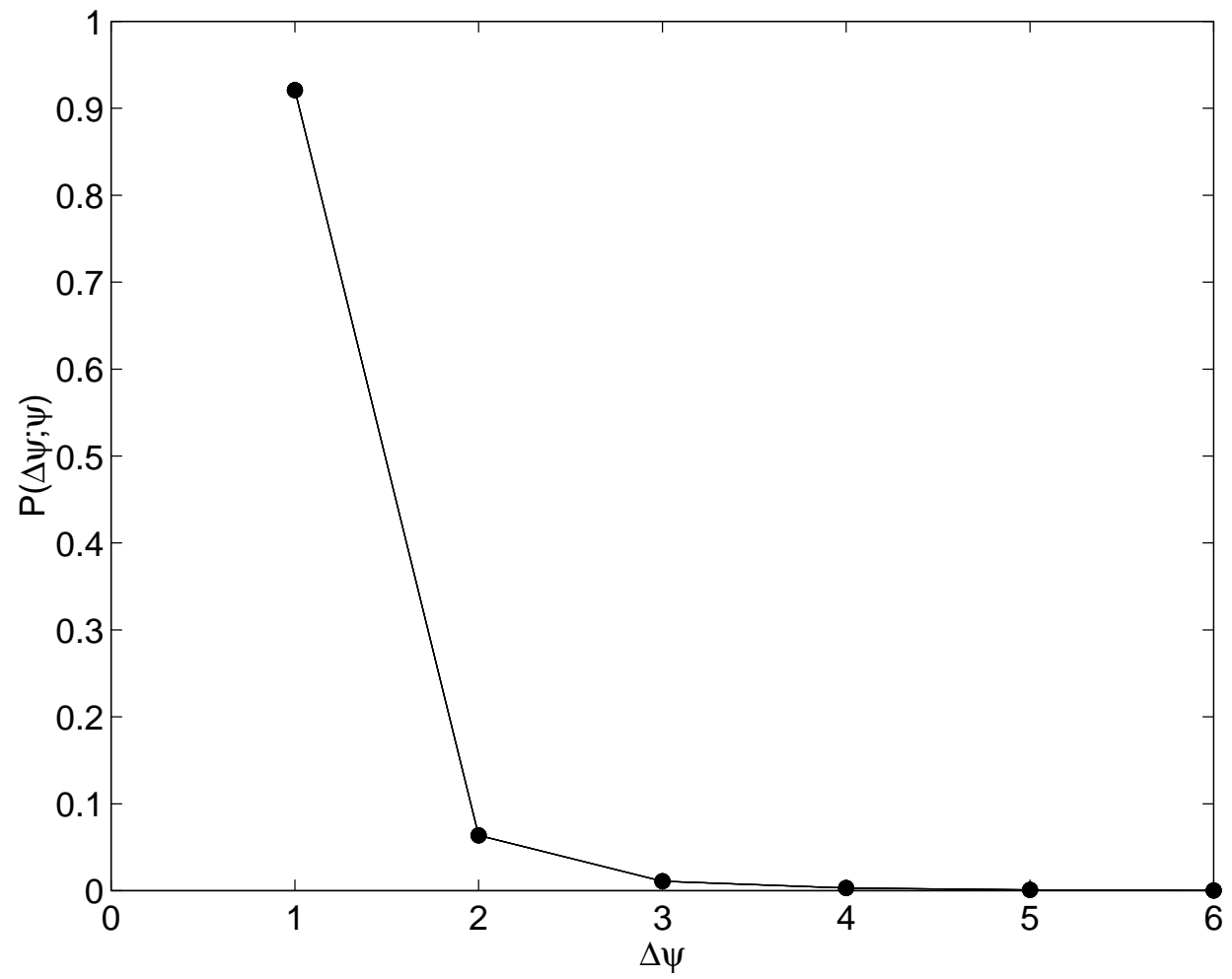

FIG. 4: Probability $P(\Delta \psi ; \psi)$ of removal (addition) of a cluster of size $\Delta \psi$ from (to) a nucleus of size $\psi=10$. This probability distribution is almost identical for all nuclei sizes $\psi \geq 10$. 


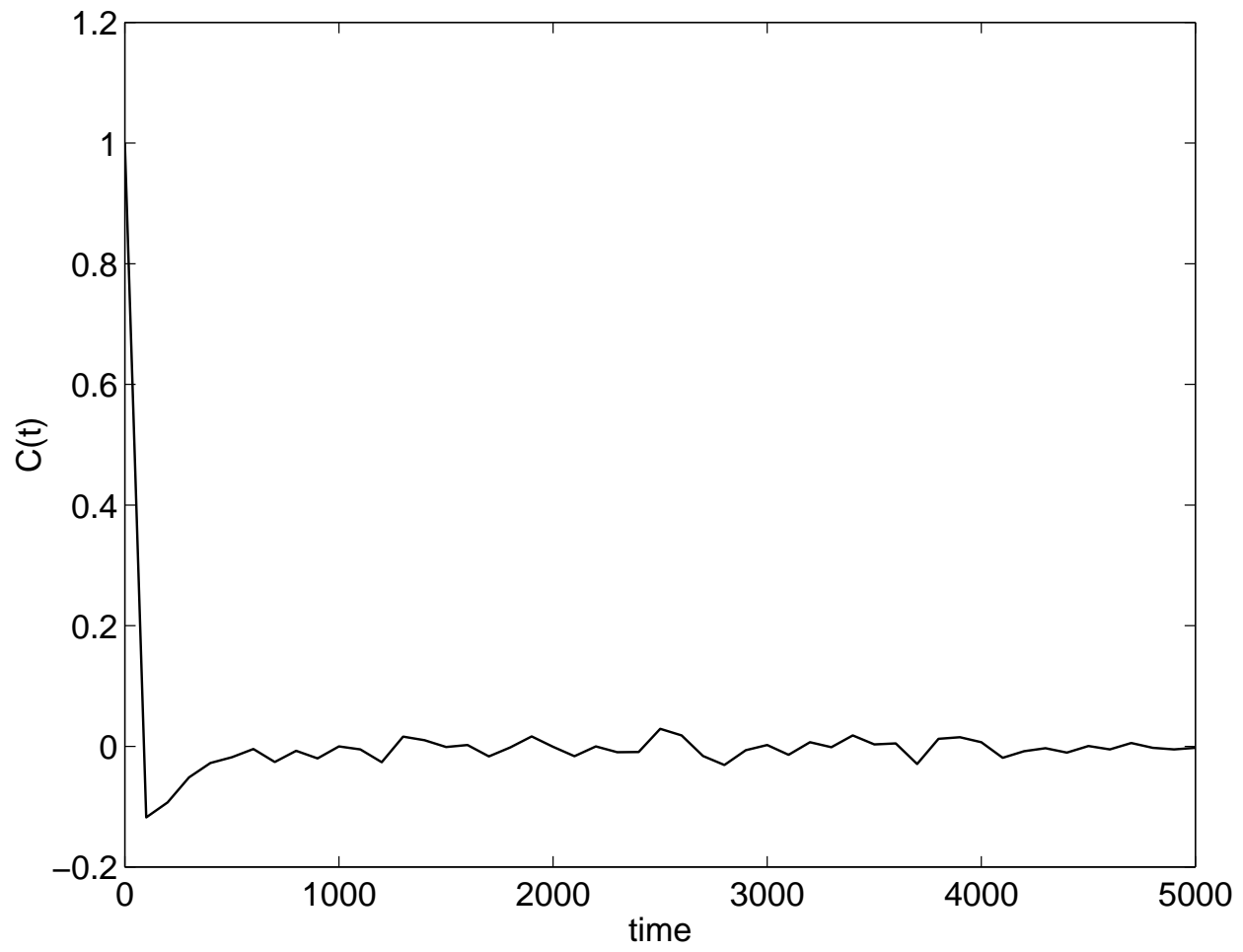

FIG. 5: Autocorrelation function $C(t)$ of the stochastic force $F(t)$; this function is normalized so that $C(0)=1$. The shown function is computed for the initial nucleus size $\psi_{0}=12$ and is typical for all $\psi_{0} \geq 10$. 

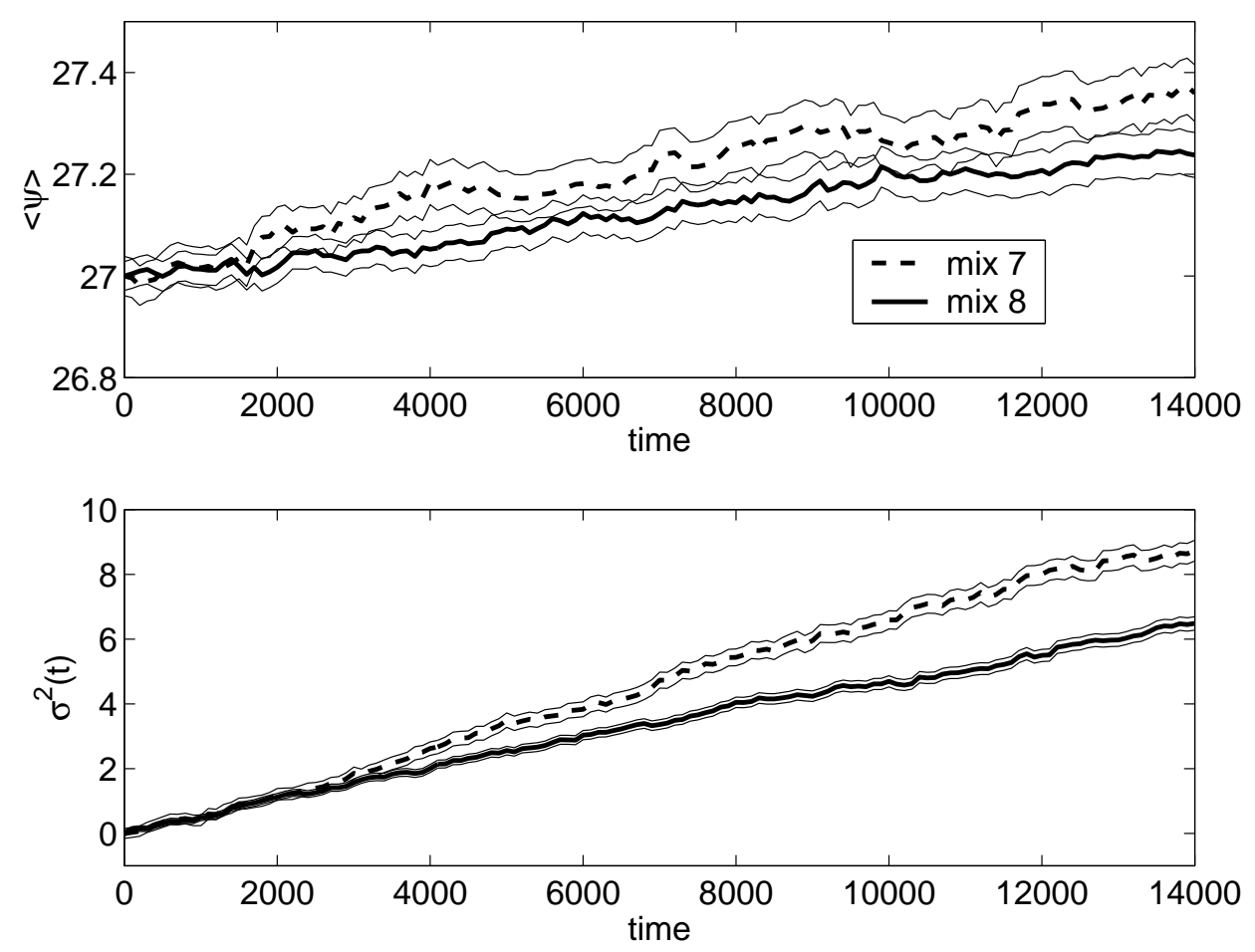

FIG. 6: Comparison of two short-scale simulations with different mixes of MC rules (mix 7 and mix 8) and the same initial nucleus size $\psi_{0}=27$. The error bars are shown by the thin lines. 


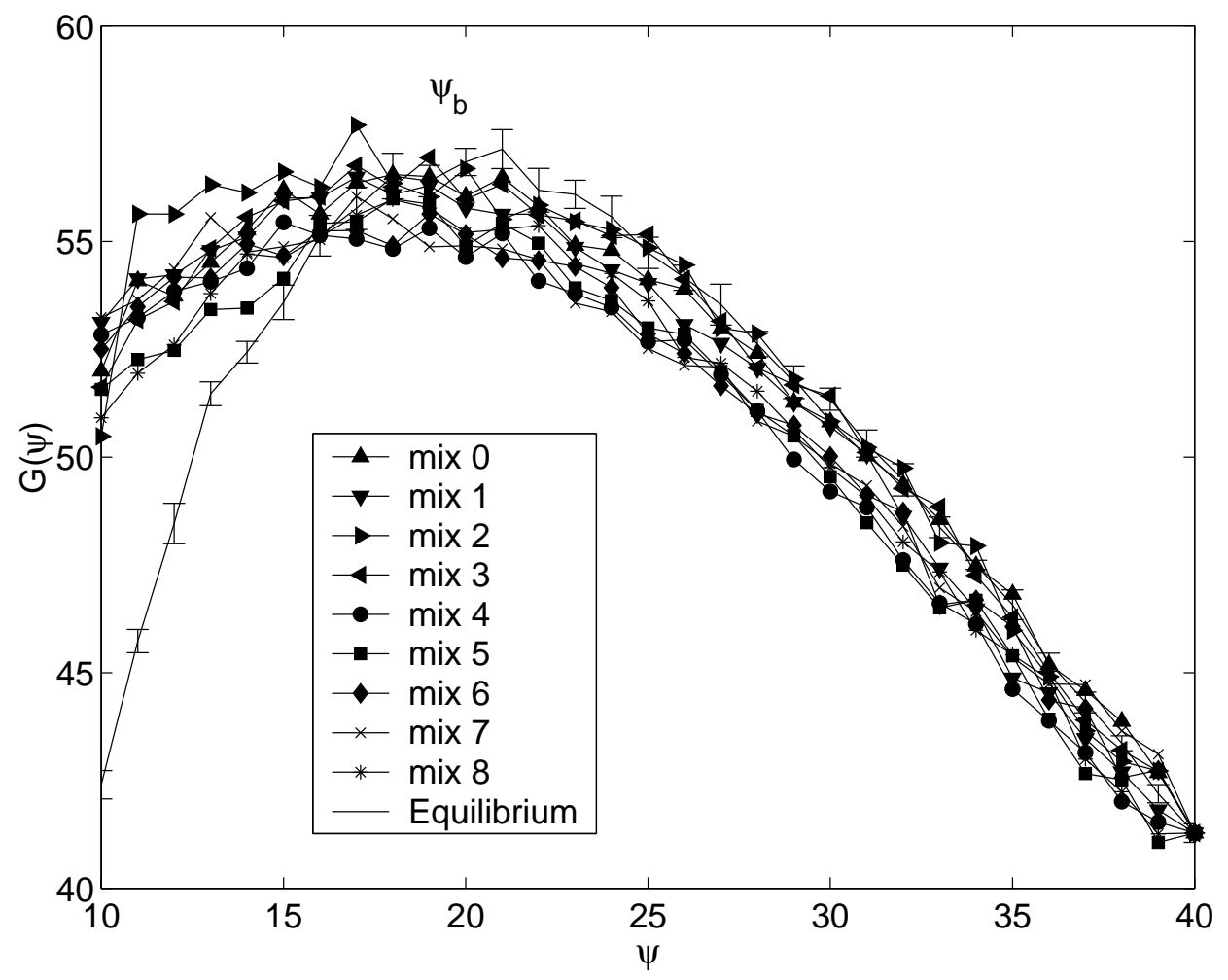

FIG. 7: Free energy $G(\psi)$ obtained from short-scale kinetic approach MC simulations with different mixes of MC moves (see Table 1 . 


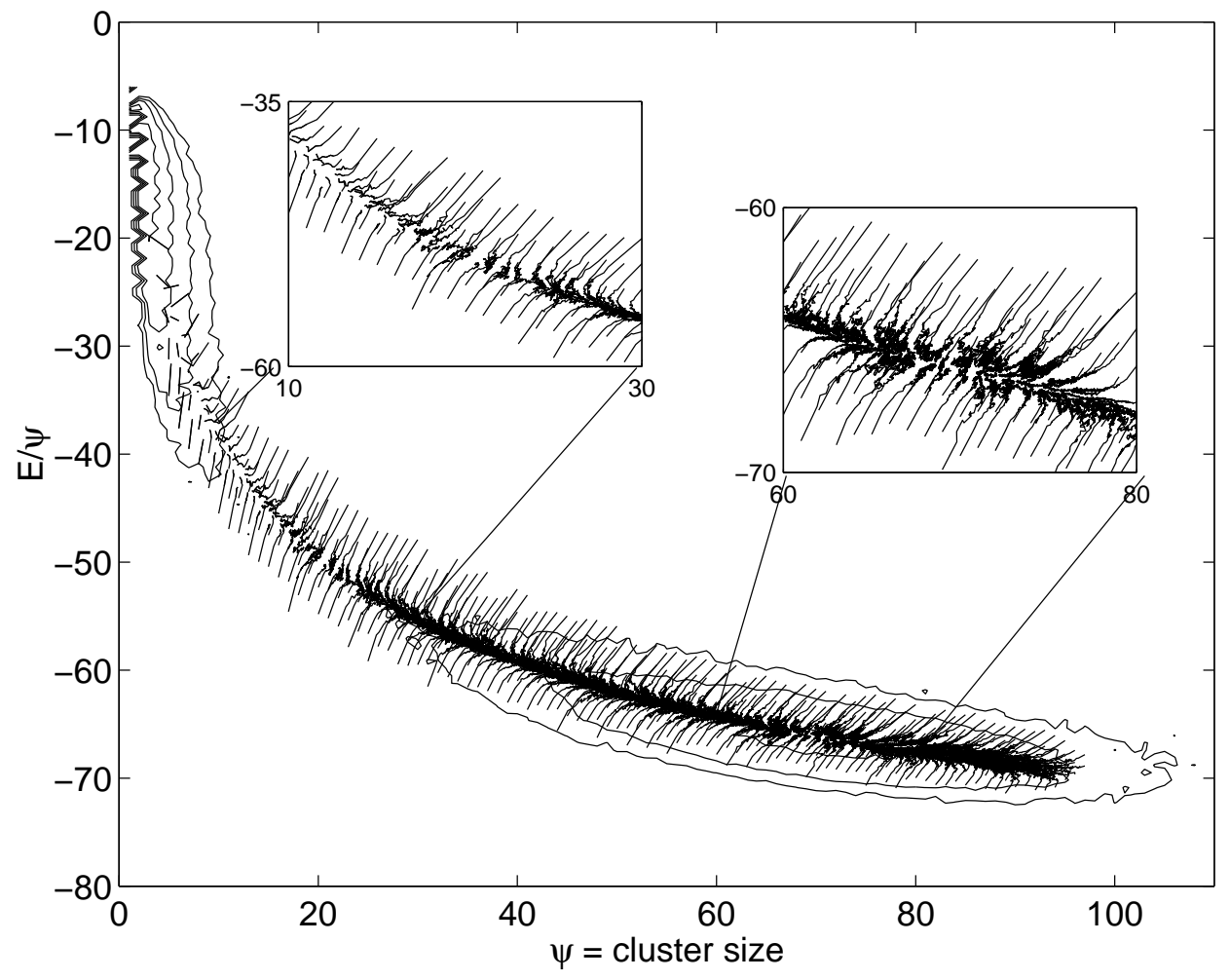

FIG. 8: Average trajectories in the $\psi-E / \psi$ phase space. Contour plot of the equilibrium free energy $G(\psi, E / \psi)$ is also shown. The insets show detailed averaged dynamics near the saddle point and the minimum of the free energy surface. 

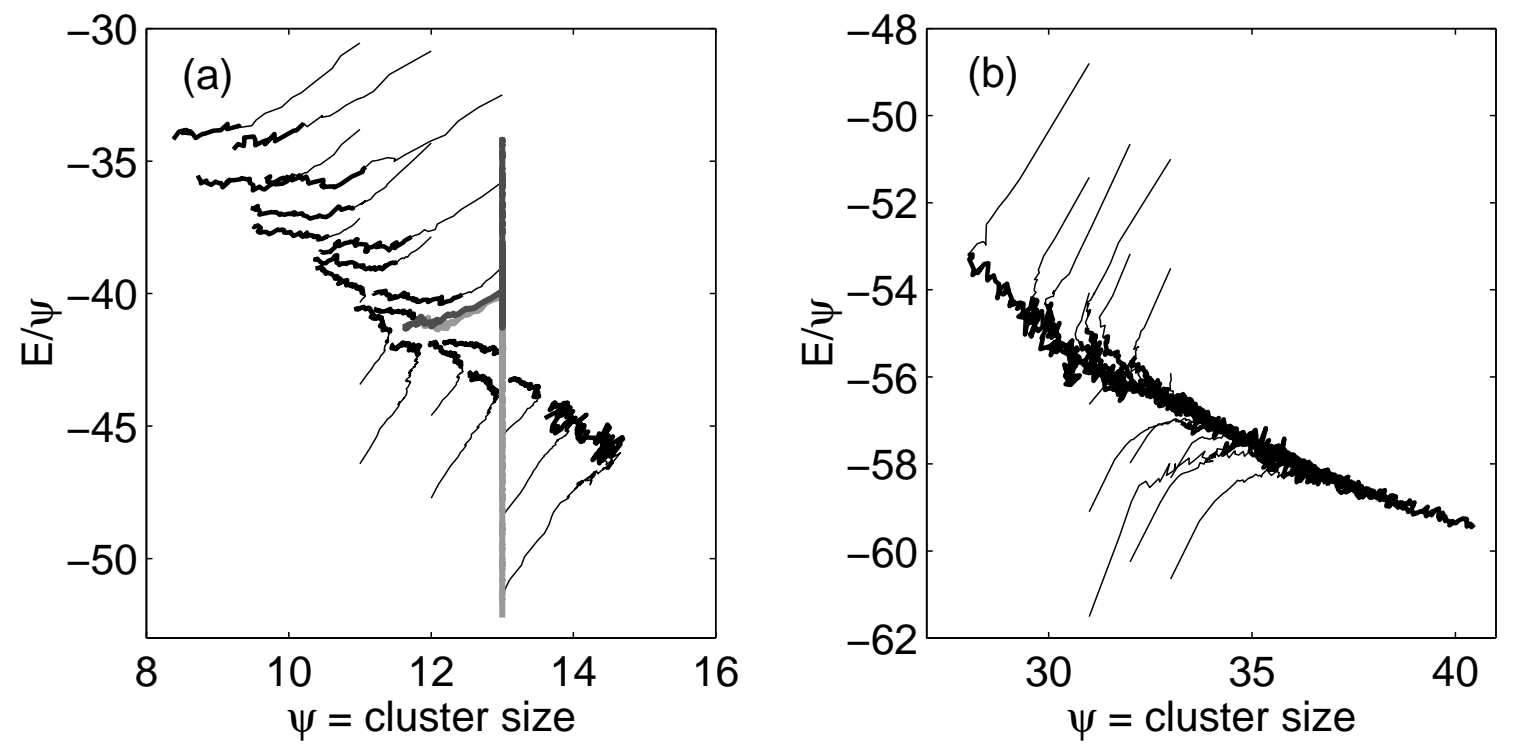

FIG. 9: Average phase trajectories for (a) $\psi_{0}=11, \ldots, 13$ (on the left of the free energy barrier $\left.\psi_{b}=21\right)$ and $(\mathrm{b}) \psi_{0}=31, \ldots, 33$ (on the right of the barrier). Thin lines correspond to $t<t_{1}$ and thick lines correspond to $t_{1}<t<t_{2}$ (see Fig. 2). In plot (a), gray lines show results of simulations with the constrained at $\psi=13$ and then released cluster size. 

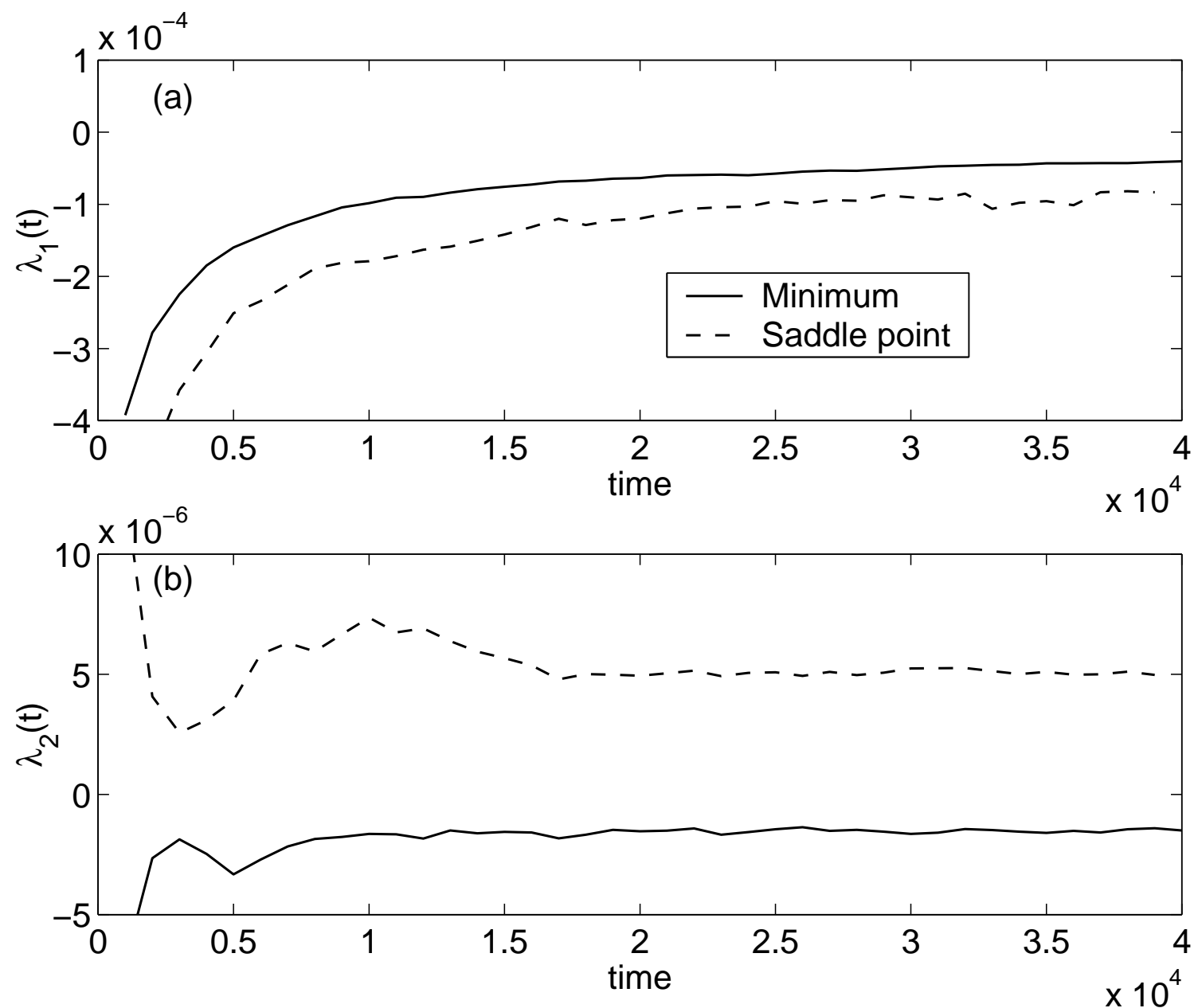

FIG. 10: Eigenvalues near the minimum (solid lines) and the saddle point (dashed lines): (a) fast eigenvalues $\lambda_{1}$ which characterize timescale of motion towards the one-dimensional manifold and (b) slow eigenvalues $\lambda_{2}$ which characterize motion along this manifold. 

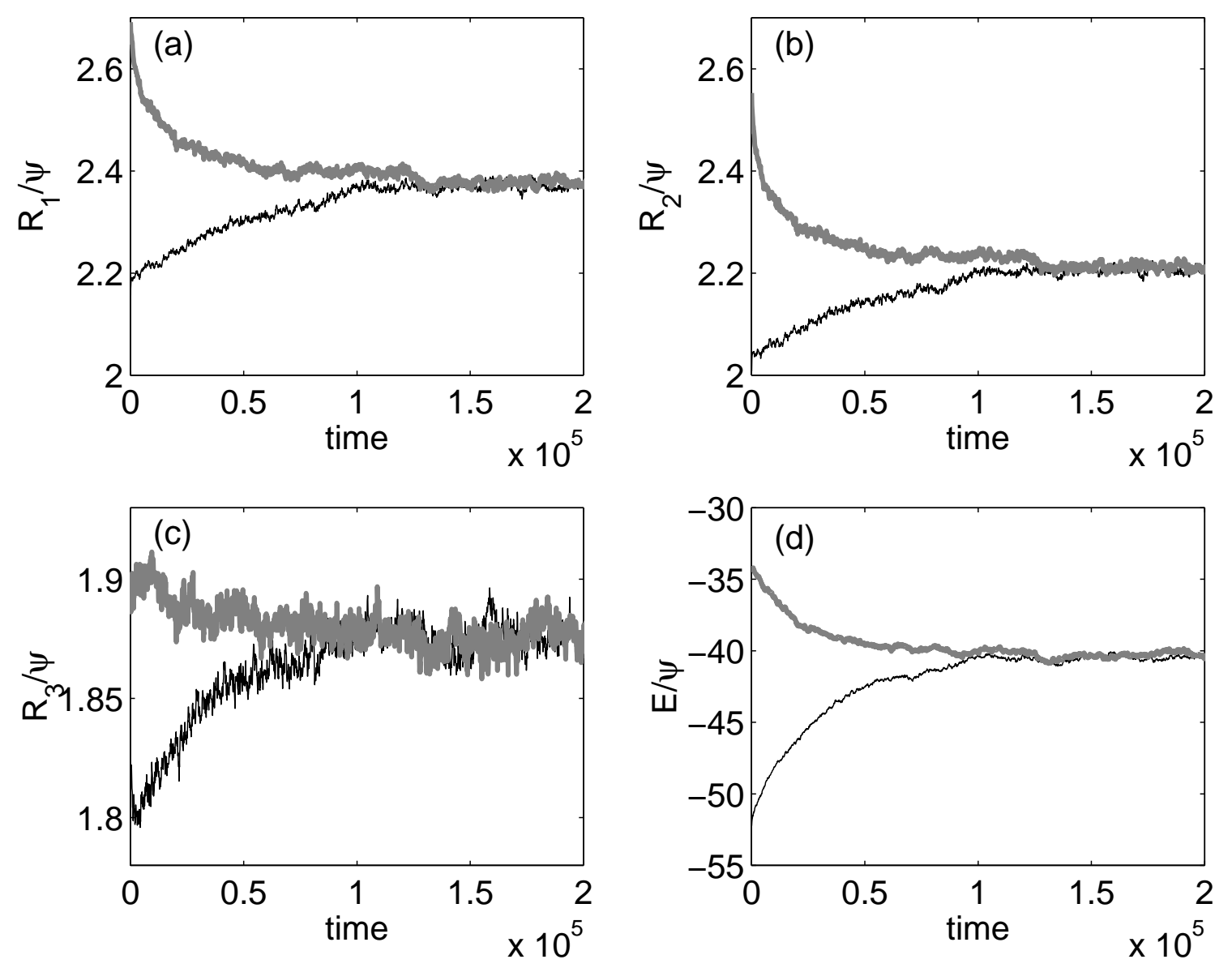

FIG. 11: Results of simulations with the cluster size constrained at $\psi=13$. (a) through (c): 1st, 2nd, and 3rd largest normalized radii of gyration; (d) normalized energy; thick gray lines show the simulation started above the one-dimensional manifold and the thin black lines show the simulation started below the manifold. 


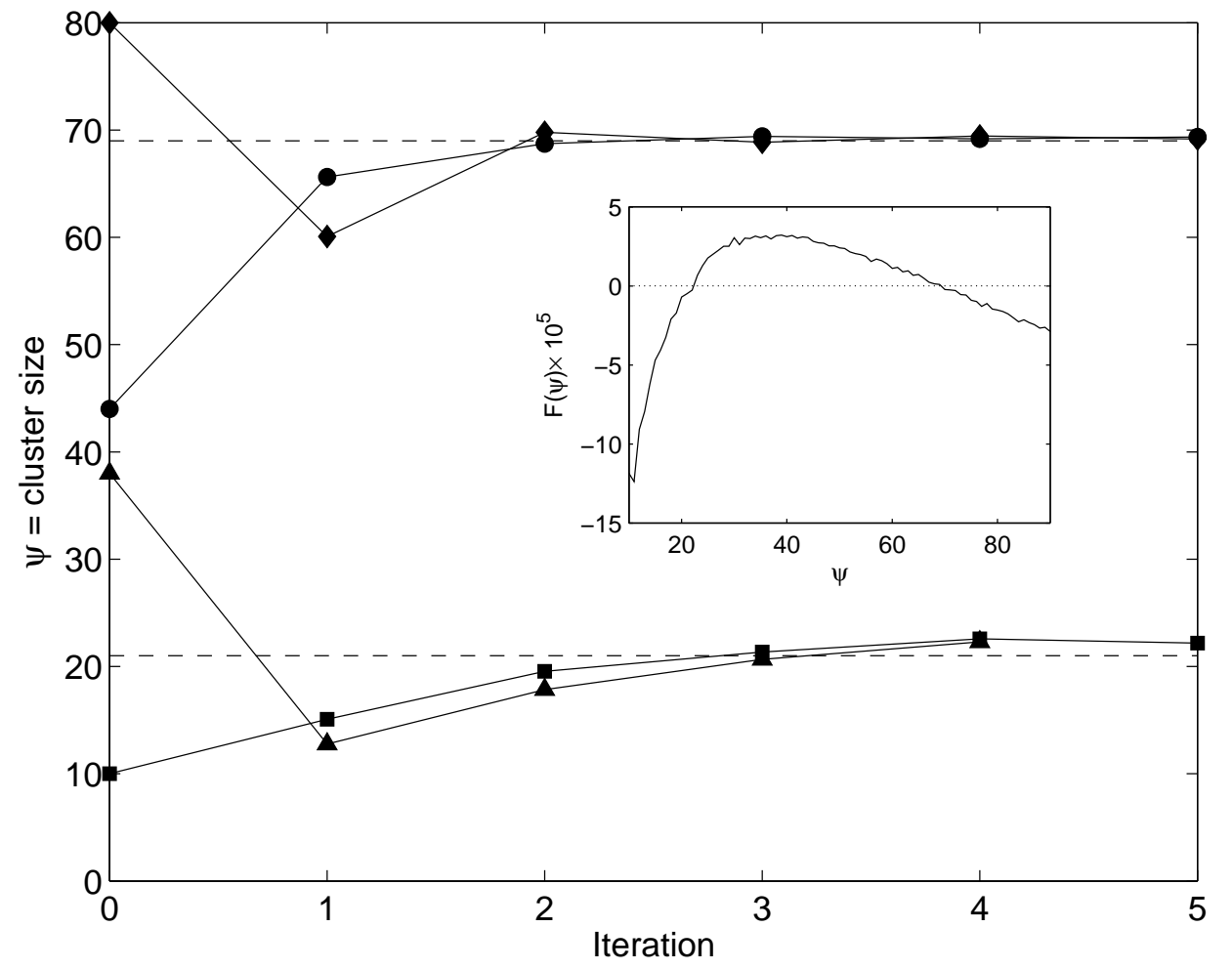

FIG. 12: Iterations of the Newton method starting from different initial conditions. Dashed lines show the minimum at $\psi_{m}=69$ and the free energy barrier at $\psi_{b}=21$. Different symbols correspond to different simulations. Solid lines are shown to guide the eye. Inset shows the function $F(\psi)$. 

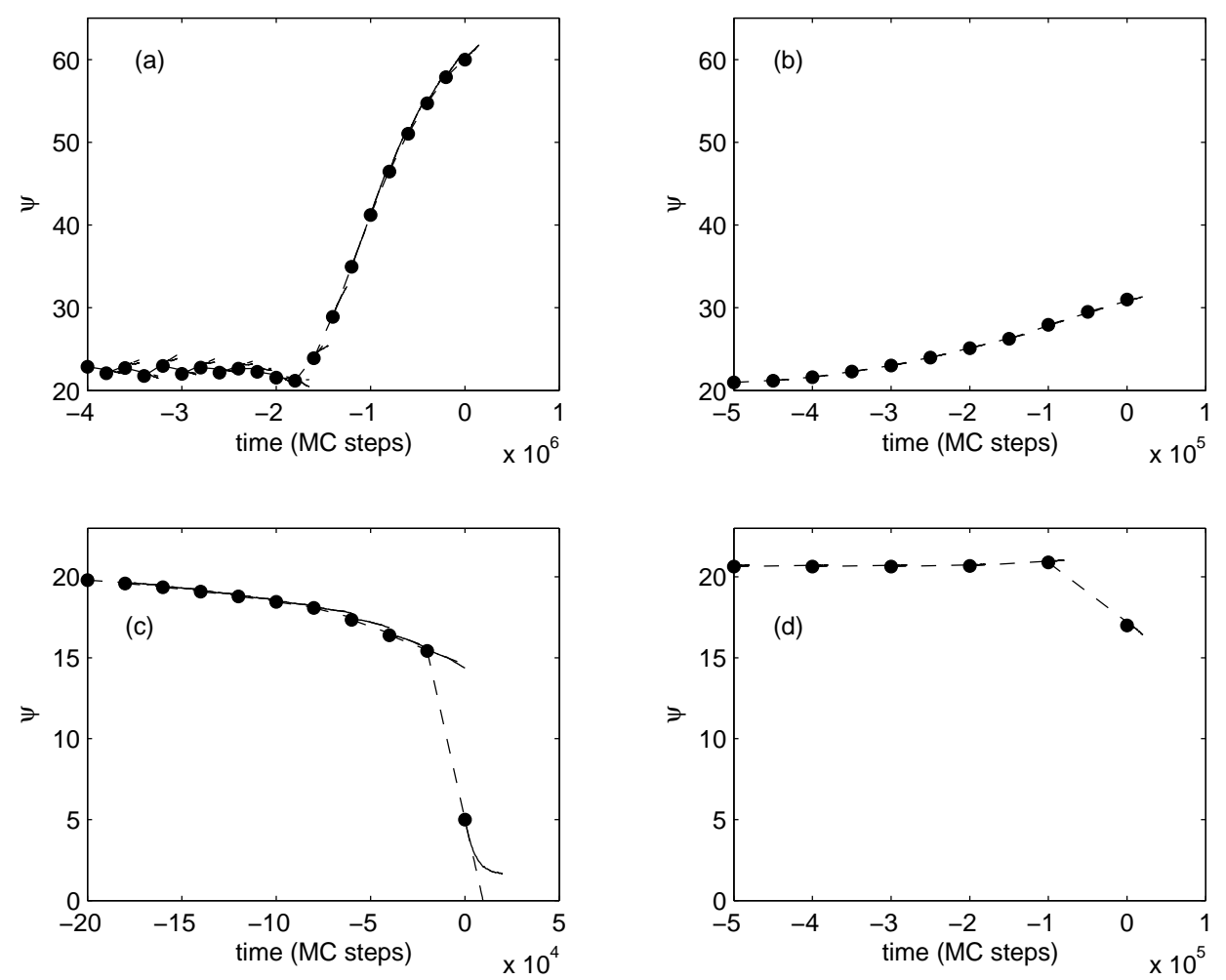

FIG. 13: Results of the coarse reverse integration: (a) initial value of the coarse variable is $\psi_{0}=60$, duration of inner simulation is $t_{\text {inner }}=2 \times 10^{5} \mathrm{MC}$ steps and the backward projection step is $h=-2 \times 10^{5} ;$ (b) $\psi_{0}=31, t_{\text {inner }}=2 \times 10^{4} \mathrm{MC}$ steps, $h=-5 \times 10^{4}$; (c) $\psi_{0}=5, t_{\text {inner }}=2 \times 10^{4}$ MC steps, $h=-2 \times 10^{4}$. (d) $\psi_{0}=17, t_{\text {inner }}=2 \times 10^{4}$ MC steps, $h=-10^{5}$. The solid lines show the short-scale forward simulation results and the dashed lines are the backward projections. The circles show the initial conditions for the short-scale simulations. 